\title{
Steady flows in unsaturated soils are stable
}

\author{
C. J. van Duijn and G. J. M. Pieters* \\ (c.j.v.duijn@tue.nl,g.j.m.pieters@tue.nl) \\ Department of Mathematics and Computer Science, \\ Eindhoven University of Technology, P.O. Box 513, 5600 MB Eindhoven, \\ The Netherlands \\ P. A. C. Raats (pac.raatsehome.nl) \\ Paaskamp 16, \\ 9301 KL Roden, The Netherlands
}

\begin{abstract}
The stability of steady, vertically upward and downward flow of water in a homogeneous layer of soil is analyzed. Three equivalent dimensionless forms of the Richards equation are introduced, namely the pressure head, saturation, and matric flux potential forms. To illustrate general results and derive special results, use is made of several representative classes of soils. For all classes of soils with a Lipschitz continuous relationship between the hydraulic conductivity and the matric flux potential, steady flows are shown to be unique. In addition, linear stability of these steady flows is proved. To this end, use is made of the energy method, in which one considers (weighted) $L^{2}$-norms of the perturbations of the steady flows. This gives a general restriction of the dependence of the hydraulic conductivity upon the matric flux potential, yielding linear stability and exponential decay with time of a specific weighted $L^{2}$-norm. It is shown that for other norms the ultimate decay towards the steadysolution is preceded by transient growth. An extension of the Richards equation to take into account dynamic memory effects is also considered. It is shown that the stability condition for the standard Richards equation implies linear stability of the steady solution of the extended model.
\end{abstract}

Keywords: Richards' equation, steady flow, uniqueness, linear stability analysis, energy method, dynamic memory effects, transient growth

\section{Introduction}

About 70 years ago, Lorenzo A. Richards consolidated the efforts of previous generations of soil physicists by formulating a general, macroscopic theory for movement of water in rigid, unsaturated soils (Richards, 1931). The theory of Richards can be formulated within the framework of the modern continuum theory of mixtures, provided that one recognizes from the outset the existence of the separate solid, liquid, and gaseous phases (Raats, 1984). It can also be justified on the basis of the principles of surface tension and viscous flow at the pore scale (Miller and Miller, 1956; Whitaker, 1986). Richards theory combines the balance of mass, expressed in the equation of continuity, and of momentum, expressed in Darcy's law. The Richards

\footnotetext{
* To whom correspondence should be addressed.
} 
equation describes movement of water in unsaturated, isothermal, rigid soils, with the air pressure everywhere and always at atmospheric pressure.

Complications beyond the standard theory may arise from thermal gradients, chemical influences (density variations, limited wettability, electrical charges on solid phase balanced by a diffuse double layer in the fluid phase), mechanical aspects associated with swelling and shrinkage, finite permeability for the gaseous phase, spatial heterogeneity, and temporal changes of the soil. But even in the realm of the standard theory, nonlinearity and the hysteretic nature of the process of water retention remain challenging. In particular, observations of seemingly unstable flows raise the question whether these can be explained in the context of the standard theory or suggest that the theory be extended.

The first studies of unstable flow in porous media dealt with the displacement of oil by water in connection with water-drive processes in oil reservoirs (Engelberts and Klinkenberg, 1951) and the displacement of sugar liquors by water from columns of granular bone charcoal in the process of refining raw sugar (Hill, 1952). Numerous studies soon followed, including studies using displacement of one fluid by another in a Hele-Shaw cell, i.e. two closely spaced parallel glass sheets serving as a model of a oil reservoir (Saffman and Taylor, 1958) and in columns of layered glass particles serving as a model for the displacement of air by water in layered soils (Tabuchi, 1961). The earliest studies focussed on the stability of the interface between two fluids, using either simple physical reasoning (Hill, 1952; Tabuchi, 1961) or formal linear stability analysis (Saffman and Taylor, 1958; Chuoke et al., 1959). The latter used the surface tension of the interface between the two fluids as the damping mechanism. In the 1960s and 1970s there was a steadily growing awareness and interest in the stability of movement of water in unsaturated soils. Systematic study of the stability of the displacement of air by water during infiltration and redistribution in soils started in the early 1970s (Hill and Parlange, 1972, Raats, 1973, Parlange and Hill, 1976, Philip 1975a, 1975b) and has been pursued ever since, theoretically as well as experimentally (see recent reviews of some aspects by de Rooij (2000), Parlange et al. (2002), and Hendrickx and Flury (2001).

Raats (1973) reviewed early observations and presented some tentative explanations. He focussed on the infiltration process and extended the GreenAmpt approach pioneered by Tabuchi (1961) to discuss effects of soil crusts, vertical heterogeneity of hydraulic conductivity, air pressure build-up ahead of wetting front, hysteresis, and wettability. Generally unstable displacement of air by water arises if the pressure gradient is such that it opposes the advance of the wetting front, but less so as the front advances. Theoretical studies and observations in the laboratory and the field have shown that this may occur for infiltration at a rate less than the hydraulic conductivity at saturation, either due to limited supply of water or due to the presence of a 
surface crust, infiltration of ponded water with compression of air ahead of the wetting front, infiltration in soils with a fine textured layer overlying a coarse textured layer, infiltration in water repellent soils, and during redistribution of water following infiltration (Raats, 1973, de Rooij, 2000, Hendrickx and Flury, 2001, Parlange et al., 2002).

Philip (1975a; 1975b) analyzed the stability of the Green-Ampt model for vertical infiltration, using linear stability analysis with a macroscopic surface tension of the wetting front as a damping mechanism, thus essentially following Saffman and Taylor (1958) and Chuoke et al. (1959). Rather than applying linear stability analysis to the equation of motion of a Green-Ampt sharp interface, Diment and Watson $(1982 ; 1983 ; 1985)$ applied it directly to the Richards equation. Their numerical solution indicated infiltration to be stable (Diment and Watson, 1983). Egorov et al. (2002) confirmed this analytically for the long time, diffuse front travelling wave solution for infiltration into initially wet soil. However, for infiltration into an initially dry soil, they found the long time, sharp front travelling wave solution to be unstable for any perturbation mode. Without the surface tension damping mechanism, Saffman and Taylor (1958), Chuoke et al. (1959), and Philip (1975a; 1975b) would have reached the same conclusion for the motion of the sharp interfaces. Egorov et al. (2002) demonstrated that the extension of the Richards equation by Hassanizadeh and Gray (1990; 1993), to take into account dynamic memory effects, may provide a damping mechanism. This critically depends on the relaxation parameter involved.

Kapoor (1996) derived stability criteria for the various types of steady, vertical upward and downward flows in homogeneous, unsaturated porous media. Using the energy method, he showed that purely gravitational flows are stable. For the other types of steady, vertical flows he derived criteria for stability/instability. Based on experimental evidence that observed fingers often are long and narrow, he assumed that the vertical length scale of the perturbations is large compared to the horizontal length scale and on that basis simplified the perturbation equation. However, linear stability analysis concerns the process of initiation of the fingers and in that stage the vertical length scale of the perturbations is still small. The observed long and narrow fingers are always connected with infiltration and redistribution processes reviewed briefly above. Therefore in this paper we reconsider the problem studied by Kapoor, without ignoring the vertical gradients. Like Kapoor, we ignore possible effects of hysteresis. Our analysis will show that the vertical gradients play an essential role in the analysis. In Section 8 we will state the conclusions of Kapoor and compare them with our results.

In Section 2, we start with three equivalent formulations of the model for flow of water in unsaturated soils, namely the pressure head, water content, and matric flux potential formulations. Making the equations dimensionless by using the limiting saturated reference state and the layer thickness, we 
introduce the dimensionless Rayleigh number as the ratio of the layer thickness and the capillary length scale of the soil. To illustrate general results and derive special results, use is made of several representative classes of soils defined by specific water retention and hydraulic conductivity characteristics, i.e. the Broadbridge-White, Burgers, and Gardner classes of soils.

In Section 3 the steady background flows are analyzed. In Subsection 3.1 we prove uniqueness of steady flows for all classes of soils with a Lipschitz continuous relationship between the hydraulic conductivity and the matric flux potential and show that among these are the Broadbridge-White, Burgers, and Gardner classes of soils. The proof of the uniqueness theorem is given in Appendix A. In Subsection 3.2 we identify the various types of steady vertical flows.

Since the uniqueness theorem suggests stability of steady vertical flows, we concentrate in this paper on deriving estimates of the rate of decay of perturbations of these steady flows. In Subsection 4.1 we prove, again subject to certain requirements concerning the relationship between the hydraulic conductivity and the matric flux potential, the stability of steady vertical flows, and, moreover, show that the squared of the weighted $L^{2}$-norm of the perturbation of the matric flux potential decays exponentially with time. In Subsection 4.2, we show that the requirements for this stability theorem are fulfilled by the Broadbridge-White, Burgers, and Gardner classes of soils and the corresponding estimates of the decay rates are derived.

In Section 5 we consider estimates of the decay rate in terms of the squared of the $L^{2}$-norm of the perturbation of the saturation. First we derive such an estimate directly from the estimate in terms of the perturbation of the matric flux potential. The resulting saturation based estimate shows that transient growth may occur, except for the Burgers class of soils. However, in Section 5 it is also indicated that in some cases a direct and sharp saturation based estimate can be obtained from the linearized equation for the perturbation of the saturation. In Appendix B this is worked out in detail for the Gardner class of soils. At the end of Section 5 it is shown that the steady solutions for the Burgers class of soils are nonlinearly stable with respect to arbitrary finite-amplitude perturbations.

In Section 6 we consider an extension of the Richards equation to take into account dynamic memory effects, in a form suggested by Hassanizadeh and Gray $(1990 ; 1993)$. Using the saturation formulation, we show that linear stability of the steady solutions corresponding to the standard Richards equation implies linear stability of the steady solutions of the extended equation.

Finally in Section 7, again for the Burgers class of soils, transient growth is studied in more detail, using a norm based on the pressure head. 


\section{Problem formulation}

In this paper we use the convention that a prime $\left({ }^{\prime}\right)$ denotes differentiation with respect to the argument. We restrict ourselves to a flow domain $\Omega$ which is bounded in the horizontal plane and which has vertical boundaries between $z=0$ and $z=H$, i.e.

$$
\Omega=\left\{(x, y, z):(x, y) \in \Omega_{\perp}, 0<z<H\right\},
$$

with $z$ being the vertical coordinate taken positive downward and where $\Omega_{\perp}$ is a bounded set in $\mathbb{R}^{2}$ with a piecewise smooth boundary $\partial \Omega_{\perp}$.

Assuming the water to be incompressible, the balance of mass can be written as a volumetric balance equation

$$
\frac{\partial \theta}{\partial t}=-\nabla \cdot \mathbf{F}
$$

where $t$ is the time, $\nabla$ is the vector differential operator, $\theta$ is the volumetric water content, $\mathbf{F}=\theta \mathbf{v}$ is the volumetric flux of the water, with $\mathbf{v}$ being the velocity of the water.

The pressure head $\Psi$ of the water is defined by

$$
\Psi=\frac{\left(p_{w}-p_{g}\right)}{\gamma g}=-\frac{p^{c}}{\gamma g}
$$

where $p_{w}$ and $p_{g}$ are the pressures of the aqueous and gaseous phases, $p^{c}$ is the capillary pressure, $\gamma$ is the density of water, and $g$ is the gravitational constant. In the theory of Richards, it is assumed that the pressure of the gaseous phase is spatially uniform and constant. Furthermore, it is assumed that the pressure head $\Psi$ is a monotonically increasing function of the volumetric water content $\theta$. In this paper we ignore the generally hysteretic nature of the $\Psi(\theta)$ relationship. The volumetric water capacity $c$ is defined as

$$
c=\frac{\mathrm{d} \theta}{\mathrm{d} \Psi} .
$$

Darcy's law for the volumetric flux $\mathbf{F}$ of the water has three alternative forms:

$$
\left\{\begin{array}{l}
\mathbf{F}=-k \nabla \Psi+k \mathbf{e}_{z}, \\
\mathbf{F}=-D \nabla \theta+k \mathbf{e}_{z}, \\
\mathbf{F}=-\nabla \Phi+k \mathbf{e}_{z} .
\end{array}\right.
$$

Here, $k$ is the hydraulic conductivity, $\mathbf{e}_{z}=\nabla z$ is the unit vector field in the $z$-direction, $D=k \mathrm{~d} \Psi / \mathrm{d} \theta$ is the soil water diffusivity, and $\Phi$ is the matric flux 
potential or Kirchhoff potential defined by

$$
\Phi=\int_{-\infty}^{\Psi} k \mathrm{~d} \Psi=\int_{0}^{\theta} D \mathrm{~d} \theta .
$$

The volumetric flux $\mathbf{F}$ is the sum of a matric component $-k \nabla \Psi=-D \nabla \theta=$ $-\nabla \Phi$ and a gravitational component $k \mathbf{e}_{z}$. The matric component of the volumetric flux is given by the gradient of $\Phi$ and therefore it is appropriate to call $\Phi$ the matric flux potential. A transformation of the type (5) was given around 1880 by Kirchhoff in his lectures on heat conduction (Kirchhoff, 1894). For this reason, $\Phi$ is often called the Kirchhoff potential and the transformation from $\Psi$ and $\theta$ to $\Phi$ is then referred to as the Kirchhoff transform.

The hydraulic conductivity $k$ is a monotonically increasing function of the volumetric water content $\theta$. Based on this $k(\theta)$ relationship, we introduce

$$
v=\frac{\mathrm{d} k}{\mathrm{~d} \theta}
$$

as the kinematic wave speed of the water.

Let $\theta_{0}$ be the volumetric water content at saturation and $\theta_{r}$ the irreducible volumetric water content. Using the saturated state as the reference state, the (apparent) saturation $S$ is defined as

$$
S=\frac{\theta-\theta_{r}}{\theta_{0}-\theta_{r}},
$$

so that $S\left(\theta_{r}\right)=0 \leqslant S \leqslant S\left(\theta_{0}\right)=1$. Using the layer thickness $H$ and the saturated reference state, we redefine the variables as follows:

$$
(\mathrm{RV} 1)\left\{\begin{array} { l } 
{ \{ x , y , z \} : = \frac { \{ x , y , z \} } { H } , } \\
{ \nabla : = H \nabla , } \\
{ t : = \frac { t } { t _ { \mathrm { ref } } } = \frac { t } { H ^ { 2 } / D _ { 0 } } , } \\
{ \mathbf { F } : = \frac { \mathbf { F } } { | \mathbf { F } _ { \mathrm { ref } } | } = \frac { \mathbf { F } } { k _ { 0 } } , } \\
{ \Psi : = \frac { \Psi } { \Psi _ { \mathrm { ref } } } = \frac { \Psi } { ( \theta _ { 0 } - \theta _ { r } ) / c _ { 0 } } , } \\
{ \Phi : = \frac { \Phi } { \Phi _ { \mathrm { ref } } } = \frac { \Phi } { D _ { 0 } ( \theta _ { 0 } - \theta _ { r } ) } , }
\end{array} \quad ( \mathrm { RV } 2 ) \quad \left\{\begin{array}{l}
c:=\frac{c}{c_{0}}, \\
k:=\frac{k}{k_{0}}, \\
D=\frac{k}{c}:=\frac{D}{D_{0}}=\frac{k / k_{0}}{c / c_{0}}, \\
v:=\frac{v}{v_{0}} .
\end{array}\right.\right.
$$

Since $\Psi \rightarrow 0$ as $S \rightarrow 1$, the scaling of $\Psi$ cannot be based on its value in the reference state. Instead $\left(\theta_{0}-\theta_{r}\right) / c_{0}$ serves as the capillary length scale. The redefinitions (RV2) of $c, k, D$, and $v$ imply $c=k=D=v=1$ when $S=1$. 
In terms of these reduced variables, the volumetric mass balance and the three forms of Darcy's law become

$$
\frac{\partial S}{\partial t}=-\nabla \cdot(R \mathbf{F})
$$

and

$$
\left\{\begin{array}{l}
R \mathbf{F}=-k \nabla \Psi+R k \mathbf{e}_{z}, \\
R \mathbf{F}=-D \nabla S+R k \mathbf{e}_{z}, \\
R \mathbf{F}=-\nabla \Phi+R k \mathbf{e}_{z},
\end{array}\right.
$$

in $\Omega:=\left\{(x, y, z):(x, y) \in \Omega_{\perp}, 0<z<1\right\}$. Here $R$ denotes the dimensionless Rayleigh number defined as

$$
R=\frac{k_{0} H}{\left(\theta_{0}-\theta_{r}\right) D_{0}}=\frac{H}{\left(\theta_{0}-\theta_{r}\right) / c_{0}} .
$$

Note that the Rayleigh number $R$ is the ratio of the layer thickness and the capillary length scale $\left(\theta_{0}-\theta_{r}\right) / c_{0}$.

Substituting Equations (9) into Equation (8) gives three alternative forms of the Richards equation, namely the pressure head, saturation, and matric flux potential forms, respectively

$$
(\mathrm{RE})\left\{\begin{array}{l}
\frac{\partial S(\Psi)}{\partial t}=c(\Psi) \frac{\partial \Psi}{\partial t}=\nabla \cdot\left(k(\Psi) \nabla \Psi-R k(\Psi) \mathbf{e}_{z}\right), \\
\frac{\partial S}{\partial t}=\nabla \cdot\left(D(S) \nabla S-R k(S) \mathbf{e}_{z}\right), \\
\frac{\partial S(\Phi)}{\partial t}=\frac{1}{D(\Phi)} \frac{\partial \Phi}{\partial t}=\nabla \cdot\left(\nabla \Phi-R k(\Phi) \mathbf{e}_{z}\right) .
\end{array}\right.
$$

Remark 1. Sometimes, alternative forms of these equations are used. For instance, Kapoor (1996) writes instead of $(\mathrm{RE})_{1}$, after redefining our pressure head to his suction head $\Psi:=-\Psi$,

$$
c(-\Psi) \frac{\partial \Psi}{\partial t}=k(-\Psi) \Delta \Psi+\frac{\mathrm{d} k(-\Psi)}{\mathrm{d} \Psi} \nabla \Psi \cdot\left(\nabla \Psi+R \mathbf{e}_{z}\right) .
$$

Introducing $\bar{c}(\Psi):=c(-\Psi), \bar{k}(\Psi):=k(-\Psi)$, and $\bar{b}(\Psi):=-\frac{\mathrm{d} k(-\Psi)}{\mathrm{d} \Psi}$, we find exactly Kapoor's formulation $(3 a-b)$ :

$$
\bar{c}(\Psi) \frac{\partial \Psi}{\partial t}+\bar{b}(\Psi) \nabla \Psi \cdot\left(\nabla \Psi+R \mathbf{e}_{z}\right)-\bar{k}(\Psi) \Delta \Psi=0 .
$$




\subsection{CLASSES OF SOILS}

In the context of Richards equation, the relationships among the saturation $S$, pressure head $\Psi$, and hydraulic conductivity $k$ define the hydraulic properties of a soil. Different classes of soils have been identified with different functions approximating the physical properties. In this paper we restrict ourselves to three typical classes.

One soil class is defined by Broadbridge and White (1988),

$$
\text { (I) }\left\{\begin{array}{l}
k(S)=\frac{C-1}{C-S} S^{2}, \\
D(S)=\frac{(C-1) C}{(C-S)^{2}}, \\
\Psi(S)=1-\frac{1}{S}-\frac{1}{C} \ln \frac{C-S}{(C-1) S}, \\
\Phi(S)=\frac{(C-1) S}{C-S},
\end{array}\right.
$$

where $1<C<\infty$. This soil class is usually referred to as the versatile nonlinear model. Note that $\Psi(S)$ has an inflection point at $S=\frac{2 C}{3}$, for $1<C \leqslant \frac{3}{2}$.

We also consider the soil class defined by Clothier et al. (1981),

$$
\text { (II) }\left\{\begin{array}{l}
k(S)=S^{2}, \\
D(S)=1, \\
\Psi(S)=1-\frac{1}{S}, \\
\Phi(S)=S,
\end{array}\right.
$$

Note that with $(\mathrm{II})_{1,2}$ Equation $(\mathrm{RE})_{2}$ is Burgers equation for inviscid flows. Therefore we refer to (II) as the Burgers class of soils. For the class of Burgers soils the saturation and matric flux potential coincide. Note that the Burgers soil class corresponds to the limiting case $C \rightarrow \infty$ of the versatile nonlinear model.

Finally we consider the Gardner class of soils, where

$$
\text { (III) }\left\{\begin{array}{l}
k(S)=\frac{S^{2}}{(1-S)^{2}+S^{2},} \\
D(S)=\frac{1}{(1-S)^{2}+S^{2}}, \\
\Psi(S)=1-\frac{1}{S}, \\
\Phi(S)=\arctan (2 S-1)+\frac{\pi}{4},
\end{array}\right.
$$


Originally only the $k(\Psi)$ relationship given by $(\mathrm{III})_{1}$ was introduced to study steady upward and downward flow for this class of soils (Gardner, 1958; Raats, 1973). Note that $k(S)$ has an inflection point at $S=\frac{1}{2}$.

\section{Steady-state background flows}

It is well known that nonlinear evolution equations like (RE) may have multiple steady solutions and each of these solutions has its own stability property. Once a steady-state is qualified and quantified as being unstable, the system bifurcates from this solution to a neighboring steady solution, which is completely different from the first one. Note that this concept of instability only makes sense when multiple steady solutions exist.

Hence, the first question that needs to be answered is: has Richards' equation (RE) subject to given boundary data multiple steady solutions?

In the following subsections we show for Broadbridge-White, Burgers, and Gardner soils uniqueness and some important properties of the steady solutions of (RE). In this paper we restrict ourselves to constant boundary data, which we give in terms of the saturation:

$$
\text { (BC) }\left\{\begin{array}{l}
\left.S\right|_{z=0}=S_{\mathrm{T}} \\
\left.S\right|_{z=1}=S_{\mathrm{B}}
\end{array} \quad \text { for all } x, y \in \Omega_{\perp}, t>0,\right.
$$

and along the vertical boundary we impose $\frac{\partial S}{\partial \mathbf{n}}=\nabla S \cdot \mathbf{n}=0$ for all $t>0$.

\subsection{UNIQUENESS OF STEADY VERTICAL FLOWS}

We first note that the different formulations in (RE) are equivalent. For instance, $(\mathrm{RE})_{2}$ and $(\mathrm{RE})_{3}$ are equivalent since

$$
\Phi=f(S)=\int_{0}^{S} D(S) \mathrm{d} S \quad(D(S)>0)
$$

is strictly increasing. Hence $S=f^{-1}(\Phi)$ exists and uniqueness of solutions of $(\mathrm{RE})_{3}$ implies uniqueness of solutions of $(\mathrm{RE})_{2}$. We show here uniqueness for $(\mathrm{RE})_{3}$ because for steady flow in the $\Phi$-formulation only the gravity term is nonlinear. Thus we consider the problem

$$
(\mathrm{P} 1) \begin{cases}\nabla \cdot\left(\nabla \Phi-R \kappa(\Phi) \mathbf{e}_{z}\right)=0 & \text { in } \Omega=: \Omega_{\perp} \times(0,1), \\ \frac{\partial \Phi}{\partial \mathbf{n}}=0 & \text { on } \partial \Omega_{\perp} \times(0,1), \\ \Phi=\Phi_{\mathrm{BC}}=f\left(S_{\mathrm{BC}}\right) & \text { on } \Omega_{\perp} \times(\{0\} \cup\{1\}),\end{cases}
$$


where $\kappa(\Phi):=k(S(\Phi)), \frac{\partial \Phi}{\partial \mathbf{n}}=\nabla \Phi \cdot \mathbf{n}$, with $\mathbf{n}$ the outward normal at $\partial \Omega_{\perp} \times$ $(0,1)$, and where $S_{\mathrm{BC}}$ denotes boundary conditions $(\mathrm{BC})$. In Appendix A we prove the following general statement:

Theorem 1. Let $M:=f(1)$ and let $\kappa(\Phi):[0, M] \rightarrow[0, \infty)$ be Lipschitz continuous: i.e. there exists a constant $L>0$ such that

$$
\left|\kappa\left(\Phi_{1}\right)-\kappa\left(\Phi_{2}\right)\right| \leqslant L\left|\Phi_{1}-\Phi_{2}\right|,
$$

for all $0 \leqslant \Phi_{1}, \Phi_{2} \leqslant M$. Then problem (P1) has a unique solution for each $R>0$. It is given by the one-dimensional profile $\Phi_{0}=\Phi_{0}(z)$ satisfying

$$
(\mathrm{P} 2)\left\{\begin{array}{l}
\frac{\mathrm{d}}{\mathrm{d} z}\left(\frac{\mathrm{d} \Phi_{0}}{\mathrm{~d} z}-R \kappa\left(\Phi_{0}\right)\right)=0 \quad \text { for } 0<z<1, \\
\Phi_{0}(0)=f\left(S_{\mathrm{T}}\right)=: \Phi_{\mathrm{T}}, \\
\Phi_{0}(1)=f\left(S_{\mathrm{B}}\right)=: \Phi_{\mathrm{B}} .
\end{array}\right.
$$

For Broadbridge-White soils the matric flux potential is given by $(\mathrm{I})_{4}$. Hence $S=f^{-1}(\Phi)=\frac{C \Phi}{\Phi+C-1}$ implying $\kappa(\Phi)=\frac{C \Phi^{2}}{\Phi+C-1}$ with $0 \leqslant \Phi \leqslant M=1$. This function is Lipschitz continuous since

$$
\begin{aligned}
\left|\kappa\left(\Phi_{1}\right)-\kappa\left(\Phi_{2}\right)\right| & =\left|\frac{C\left(\Phi_{1} \Phi_{2}+\left(\Phi_{1}+\Phi_{2}\right)(C-1)\right)\left(\Phi_{1}-\Phi_{2}\right)}{\left(\Phi_{1}+C-1\right)\left(\Phi_{2}+C-1\right)}\right| \leqslant \\
& \leqslant \frac{C(2 C-1)}{(C-1)^{2}}\left|\Phi_{1}-\Phi_{2}\right|=: L(C)\left|\Phi_{1}-\Phi_{2}\right|,
\end{aligned}
$$

for all $0 \leqslant \Phi_{1}, \Phi_{2} \leqslant 1$. For $1<C<\infty$ we have $2<L(C)<\infty$. Hence the steady solutions for the Broadbridge-White class of soils, including the Burgers class of soils as a limiting special case, are unique.

For Gardner class soils the matric flux potential is given by $(\mathrm{II})_{4}$. Hence $S=f^{-1}(\Phi)=\frac{1}{1+\cot (\Phi)}$, implying $\kappa(\Phi)=\sin ^{2}(\Phi)$ for $0 \leqslant \Phi \leqslant M=\frac{\pi}{2}$. Hence

$$
\left|\kappa\left(\Phi_{1}\right)-\kappa\left(\Phi_{2}\right)\right|=\frac{1}{2}\left|\cos \left(2 \Phi_{1}\right)-\cos \left(2 \Phi_{2}\right)\right| \leqslant\left|\Phi_{1}-\Phi_{2}\right|,
$$

for all $0 \leqslant \Phi_{1}, \Phi_{2} \leqslant \frac{\pi}{2}$. We conclude that the steady solutions for the Gardner class of soils are also unique.

The theorem rules out any other stationary solution satisfying the boundary conditions. In particular finger-like solutions, describing steady convection cells, do not exist. The theorem also suggests that problem (P2) describes the large time behaviour of transient solutions for any $R>0$. With the exception of chaotic or temporally periodic behaviour, what else could be possible large time behaviour? In the next section we show that indeed problem $(\mathrm{P} 2)$ describes the large time behaviour and we give rates of convergence. 
3.2. SOME PROPERTIES OF PROBLEM (P2)

To obtain the steady solution, we integrate $(\mathrm{P} 2)_{1}$ with respect to $z$, i.e.

$$
\frac{\mathrm{d} \Phi_{0}}{\mathrm{~d} z}=R\left\{\kappa\left(\Phi_{0}\right)-F\right\},
$$

where $R F$ denotes the flux. The solutions of (13) subject to $(\mathrm{P} 2)_{2}$ and $(\mathrm{P} 2)_{3}$, can be classified as follows (see also Figure 1):

The case $\Phi_{\mathrm{T}}>\Phi_{\mathrm{B}}$, or equivalently $S_{\mathrm{T}}>S_{\mathrm{B}}$.

(1) Downward flow aided by capillarity: for this case $\Phi_{0}^{\prime}(z)<0$. From (13), using the fact that $\kappa\left(\Phi_{0}\right)$ is a monotonically increasing function, and since $R>0$ we find $F>\kappa\left(\Phi_{\mathrm{T}}\right) \geqslant \kappa\left(\Phi_{0}\right)$.

The case $\Phi_{\mathrm{T}} \equiv \Phi_{\mathrm{B}}$, or equivalently $S_{\mathrm{T}} \equiv S_{\mathrm{B}}$.

(2) Purely gravitational downward flow: for this case we have $\Phi_{0}^{\prime}(z) \equiv 0$. From (13) it then follows that $F=\kappa\left(\Phi_{\mathrm{T}}\right)=\kappa\left(\Phi_{\mathrm{B}}\right)$.

The case $\Phi_{\mathrm{T}}<\Phi_{\mathrm{B}}$, or equivalently $S_{\mathrm{T}}<S_{\mathrm{B}}$.

(3) Downward flow opposed by capillarity: we now have $\Phi_{0}^{\prime}(z)>0$. By again using (13), the monotonicity of $\kappa\left(\Phi_{0}\right)$, and since we still deal with a downward flow, we obtain $0<F<\kappa\left(\Phi_{T}\right) \leqslant \kappa\left(\Phi_{0}\right)$. These flows only exist when $\Phi_{\mathrm{T}}>0$.

(4) Equilibrium: for this case $F \equiv 0$. Note that this case is only possible when $S_{\mathrm{T}}<S_{\mathrm{B}}$. From (13) we derive $\Phi_{0}^{\prime}(z)=R \kappa\left(\Phi_{0}\right)$.

(5) Upward flow: now we have $F<0$ and $\Phi_{0}^{\prime}(z)>R \kappa\left(\Phi_{0}\right)>0$.

Separation of variables in (13) yields

$$
R z=\int_{\Phi_{\mathrm{T}}}^{\Phi_{0}(z)} \frac{1}{\kappa\left(\Phi_{0}\right)-F} \mathrm{~d} \Phi_{0} .
$$

The solution strategy is as follows. For given boundary condition $\Phi_{\mathrm{T}}$ and Rayleigh number $R>0$, we solve (14). Here $F$ is still unknown. It has to be chosen such that the second boundary condition $\Phi=\Phi_{\mathrm{B}}$ is satisfied. Again using (14), such a $F$ can be found by inverting

$$
R=R(F)=\int_{\Phi_{\mathrm{T}}}^{\Phi_{\mathrm{B}}} \frac{1}{\kappa\left(\Phi_{0}\right)-F} \mathrm{~d} \Phi_{0} .
$$

A typical $R(F)$ relation is depicted in Figure 1. Note that $R(F)$ is a strictly monotonic decreasing (when $\Phi_{\mathrm{T}}>\Phi_{\mathrm{B}}$ ) or increasing (when $\Phi_{\mathrm{T}}<\Phi_{\mathrm{B}}$ ) function and for every $R>0$ we find a unique $F$ and hence a steady state. 


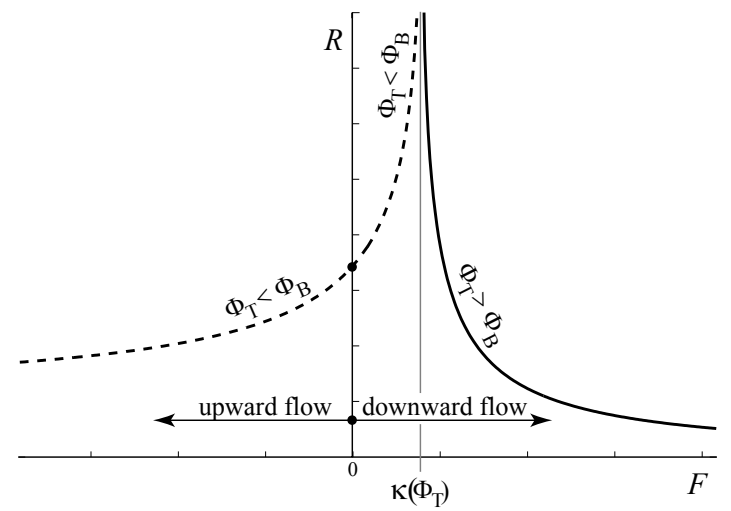

Figure 1. The Rayleigh number $R$ as function of $F$.

\section{Transient behaviour of perturbations of the matric flux potential}

\subsection{GENERAL APPROACH}

In this section we consider transient solutions $\Phi=\Phi(x, y, z, t)$ of $(\mathrm{RE})_{3}$ in $\Omega$ that satisfy the boundary conditions $\Phi_{\mathrm{T}}$ at $\{z=0\}, \Phi_{\mathrm{B}}$ at $\{z=1\}, \nabla \Phi \cdot \mathbf{n}=0$ along the vertical boundaries, and some initial condition $\left.\Phi\right|_{t=0}=\Phi(x, y, z)$. In particular, we investigate the stability of the steady solution $\Phi_{0}$. For this purpose we write

$$
\Phi=\Phi_{0}(z)+\varphi,
$$

where the perturbation $\varphi=\varphi(x, y, z, t)$ vanishes at the top $(z=0)$ and bottom $(z=1)$ of the flow domain and satisfies $\nabla \varphi \cdot \mathbf{n}=0$ along $\partial \Omega_{\perp} \times(0,1)$.

We substitute (16) into $(\mathrm{RE})_{3}$ and disregard nonlinear terms in $\varphi$. Thus we investigate linearized stability. A common way, e.g. see Egorov et al. (2003), is to investigate the spectrum of the linearized operator. Here we follow a different route. We are going to estimate the weighted $L^{2}$-norm of the perturbation $\varphi$. We prove that this norm is decreasing in time and we give an estimate of the rate of convergence.

We assume here that $\kappa:[0, M] \rightarrow[0, \infty)$ is a smooth function satisfying $\kappa(0)=0, \kappa(M)=1$, and $\kappa^{\prime}(\Phi) \geqslant 0$ for $0 \leqslant \Phi \leqslant M$. Further, $\kappa$ is possible convexconcave: i.e. there exists $0<\widehat{M} \leqslant M$ such that

$$
\kappa^{\prime \prime}(\Phi)= \begin{cases}\geqslant 0 & \text { for } 0 \leqslant \Phi<\widehat{M}, \\ <0 & \text { for } \widehat{M}<\Phi \leqslant M .\end{cases}
$$

With respect to $S(\Phi)$ we assume

$$
0<K_{1} \leqslant S^{\prime}(\Phi)=\frac{1}{D(\Phi)} \leqslant K_{2}<\infty \quad \text { for } 0 \leqslant \Phi \leqslant M .
$$


Then we prove the following stability result:

Theorem 2. Let $m_{*}:=\min \left\{\Phi_{\mathrm{T}}, \widehat{M}\right\}, m^{*}:=\max \left\{\Phi_{\mathrm{T}}, \widehat{M}\right\}$, and let

$$
\lambda:= \begin{cases}\frac{1}{2} \int_{0}^{m_{*}} \frac{\kappa^{\prime}\left(m_{*}\right)-\kappa^{\prime}(\phi)}{\kappa\left(m_{*}\right)-\kappa(\phi)} \mathrm{d} \phi \quad \text { for } S_{\mathrm{T}}>S_{\mathrm{B}}\left(\operatorname{or} \Phi_{\mathrm{T}}>\Phi_{\mathrm{B}}\right), \\ -\frac{1}{2} \int_{m^{*}}^{M} \frac{\kappa^{\prime}\left(m^{*}\right)-\kappa^{\prime}(\phi)}{\kappa\left(m^{*}\right)-\kappa(\phi)} \mathrm{d} \phi & \text { for } S_{\mathrm{T}}<S_{\mathrm{B}}\left(\operatorname{or} \Phi_{\mathrm{T}}<\Phi_{\mathrm{B}}\right) .\end{cases}
$$

Further we consider the weighted $L^{2}$-norm

$$
\|\varphi(t)\|^{2}:=\int_{\Omega} S^{\prime}\left(\Phi_{0}(x, y, z)\right) \varphi^{2}(x, y, z, t) \mathrm{d} x \mathrm{~d} y \mathrm{~d} z \quad \text { for } t \geqslant 0 .
$$

If $\lambda<1$, then

(A) $\|\varphi(t)\|^{2}$ is strictly decreasing for $t>0$,

and in particular

(B) $\|\varphi(t)\|^{2} \leqslant \mathrm{e}^{-2(1-\lambda) t / K_{2}}\|\varphi(0)\|^{2}$ for all $t>0$.

Hence if $\lambda<1$, the steady solution $\Phi_{0}$ is linearly exponentially stable.

Proof. Substitution of (16) in $(\mathrm{RE})_{3}$ and linearizing the resulting equation gives for the perturbation $\varphi$

$$
S^{\prime}\left(\Phi_{0}\right) \frac{\partial \varphi}{\partial t}=\Delta \varphi-R \frac{\partial}{\partial z}\left(\kappa^{\prime}\left(\Phi_{0}\right) \varphi\right) .
$$

To obtain estimates from (20) we use the energy method (Straughan, 1992). Multiplying (20) by $\varphi$ and integrating the equation over $\Omega$ gives

$$
\frac{1}{2} \frac{\mathrm{d}}{\mathrm{d} t}\left\|\varphi^{2}(t)\right\|^{2}=-\int_{\Omega}|\nabla \varphi|^{2}-R \int_{\Omega} \frac{\partial}{\partial z}\left(\kappa^{\prime}\left(\Phi_{0}\right) \varphi\right) \varphi,
$$

Integration by parts of the last term in (21) and using the condition that $\varphi$ vanishes along the horizontal boundaries gives

$$
\begin{aligned}
\frac{1}{2} \frac{\mathrm{d}}{\mathrm{d} t}\left\|\varphi^{2}(t)\right\|^{2} & =-\int_{\Omega}|\nabla \varphi|^{2}+\frac{R}{2} \int_{\Omega} \kappa^{\prime}\left(\Phi_{0}\right) \frac{\partial \varphi^{2}}{\partial z}= \\
& =-\int_{\Omega}|\nabla \varphi|^{2}-\frac{R}{2} \int_{\Omega}\left(\kappa^{\prime \prime}\left(\Phi_{0}\right) \frac{\mathrm{d} \Phi_{0}}{\mathrm{~d} z}\right) \varphi^{2} .
\end{aligned}
$$

Let $S_{\mathrm{T}}>S_{\mathrm{B}}$. Then $\frac{\mathrm{d} \Phi_{0}}{\mathrm{~d} z}<0$. Using this monotonicity and (17) gives

$$
\frac{1}{2} \frac{\mathrm{d}}{\mathrm{d} t}\left\|\varphi^{2}(t)\right\|^{2} \leqslant-\int_{\Omega}|\nabla \varphi|^{2}-\frac{R}{2} \int_{\Omega_{*}}\left(\kappa^{\prime \prime}\left(\Phi_{0}\right) \frac{\mathrm{d} \Phi_{0}}{\mathrm{~d} z}\right) \varphi^{2},
$$


where $\Omega_{*}:=\left\{(x, y, z):(x, y) \in \Omega_{\perp}, z_{*}<z<1\right\}$ and $\Phi_{0}\left(z_{*}\right)=m_{*}$. To estimate the sign of the second term on the right-hand side of (22) we use the following argument. For any fixed $(x, y) \in \Omega_{\perp}$ and $t>0$ we write $\varphi(z):=\varphi(x, y, z, t)$ and estimate with Cauchy-Schwarz

$$
\begin{aligned}
\varphi(z) & \leqslant|-\varphi(z)|=\left|\int_{z}^{1} \varphi^{\prime}(\zeta) \mathrm{d} \zeta\right| \leqslant \\
& \leqslant\left(\int_{z}^{1} 1^{2} \mathrm{~d} \zeta\right)^{1 / 2}\left(\int_{z}^{1}\left(\varphi^{\prime}(\zeta)\right)^{2} \mathrm{~d} \zeta\right)^{1 / 2} \leqslant \\
& \leqslant \sqrt{1-z}\left(\int_{0}^{1}\left(\varphi^{\prime}(\zeta)\right)^{2} \mathrm{~d} \zeta\right)^{1 / 2} .
\end{aligned}
$$

Then, using Fubini's Theorem and inequality (23) in (22), we have

$$
\begin{aligned}
& \frac{1}{2} \frac{\mathrm{d}}{\mathrm{d} t}\left\|\varphi^{2}(t)\right\|^{2} \leqslant-\int_{\Omega}|\nabla \varphi|^{2}- \\
&-\frac{R}{2}\left\{\int_{z_{*}}^{1}(1-z) \frac{\mathrm{d}}{\mathrm{d} z} \kappa^{\prime}\left(\Phi_{0}\right) \mathrm{d} z\right\} \int_{\Omega}|\nabla \varphi|^{2}=: \\
&=:-(1-\lambda) \int_{\Omega}|\nabla \varphi|^{2} .
\end{aligned}
$$

For the factor $\lambda$ in (24) we obtain, using (19) and the fact that $m_{*} \leqslant \Phi_{\mathrm{T}}$,

$$
\begin{aligned}
\lambda & =-\left.\frac{R}{2}(1-z) \kappa^{\prime}\left(\Phi_{0}\right)\right|_{z_{*}} ^{1}-\frac{R}{2} \int_{z_{*}}^{1} \kappa^{\prime}\left(\Phi_{0}\right) \mathrm{d} z= \\
& =\frac{R}{2} \int_{z_{*}}^{1}\left\{\kappa^{\prime}\left(m_{*}\right)-\kappa^{\prime}\left(\Phi_{0}\right)\right\} \mathrm{d} z=\frac{1}{2} \int_{\Phi_{\mathrm{B}}}^{m_{*}} \frac{\kappa^{\prime}\left(m_{*}\right)-\kappa^{\prime}\left(\Phi_{0}\right)}{\kappa\left(\Phi_{\mathrm{T}}\right)-\kappa\left(\Phi_{0}\right)} \mathrm{d} \Phi_{0} \leqslant \\
& \leqslant \frac{1}{2} \int_{0}^{m_{*}} \frac{\kappa^{\prime}\left(m_{*}\right)-\kappa^{\prime}\left(\Phi_{0}\right)}{\kappa\left(m_{*}\right)-\kappa\left(\Phi_{0}\right)} \mathrm{d} \Phi_{0}<1
\end{aligned}
$$

by assumption. Hence, with the Poincaré inequality $\int_{\Omega} \varphi^{2} \leqslant \int_{\Omega}|\nabla \varphi|^{2}$ (Zeidler, 1995) and using (18), we obtain from (24) the estimate

$$
\frac{1}{2} \frac{\mathrm{d}}{\mathrm{d} t}\left\|\varphi^{2}(t)\right\|^{2} \leqslant-(1-\lambda) \int_{\Omega} \varphi^{2} \leqslant-\left((1-\lambda) / K_{2}\right)\left\|\varphi^{2}(t)\right\|^{2} \leqslant 0,
$$

which proves (A). Integrating (25) with respect to time $t$ gives

$$
\left\|\varphi^{2}(t)\right\|^{2} \leqslant \mathrm{e}^{-2(1-\lambda) t / K_{2}}\left\|\varphi^{2}(0)\right\|^{2},
$$

which proves $(\mathrm{B})$.

The case $S_{\mathrm{T}}<S_{\mathrm{B}}$ follows in a similar fashion. Its proof is therefore omitted. 


\subsection{APPLICATION TO SPECIFIC CLASSES OF SOILS}

\subsubsection{Broadbridge-White and Burgers classes}

First observe that $\kappa(\Phi)$ has no inflection points for $0 \leqslant \Phi \leqslant M=1$. Hence $\widehat{M} \equiv M=1$ and $m^{*} \equiv 1$. This immediately implies that $\lambda \equiv 0<1$ for the case $S_{\mathrm{T}}<S_{\mathrm{B}}$, and, hence, that in terms of the classification of the steady vertical flows in Subsection 3.2, downward flows opposed by capillarity, equilibrium, and upward flows are linearly stable.

For the case $S_{\mathrm{T}}>S_{\mathrm{B}}$, i.e. for downward flows aided by capillarity, we estimate $\lambda$. Since $0 \leqslant m_{*} \leqslant \widehat{M}=1$ we have

$$
\begin{aligned}
\lambda & =\frac{1}{2} \int_{0}^{m_{*}} \frac{\kappa^{\prime}\left(m_{*}\right)-\kappa^{\prime}(\phi)}{\kappa\left(m_{*}\right)-\kappa(\phi)} \mathrm{d} \phi= \\
& =\frac{1}{2}\left[\ln \left(\frac{2 C-2+m_{*}}{C-1+m_{*}}\right)+\left(\frac{C-1}{C-1+m_{*}}\right)^{2} \ln \left(\frac{2 C-2+m_{*}}{C-1}\right)\right] \leqslant \ln 2
\end{aligned}
$$

for all $C>1$. Hence $\lambda \leqslant \ln 2<1$ and this implies that $\Phi_{0}$ is linearly stable.

\subsubsection{Gardner class}

Observe that for this case $\kappa(\Phi)$ has an inflection point at $\Phi=\frac{\pi}{4}$. This implies $\widehat{M}=\frac{\pi}{4}$. We first consider the case $S_{\mathrm{T}}>S_{\mathrm{B}}$. Since $0 \leqslant \phi \leqslant m_{*} \leqslant \frac{\pi}{4}$ we have

$$
\begin{aligned}
\lambda & =\frac{1}{2} \int_{0}^{m_{*}} \frac{\kappa^{\prime}\left(m_{*}\right)-\kappa^{\prime}(\phi)}{\kappa\left(m_{*}\right)-\kappa(\phi)} \mathrm{d} \phi= \\
& =\frac{1}{2} \int_{0}^{m_{*}} \frac{\left(\sin \left(2 m_{*}\right)-\sin (2 \phi)\right)\left(\phi+m_{*}\right)}{\sin ^{2}\left(m_{*}\right)-\sin ^{2}(\phi)} \frac{1}{\phi+m_{*}} \mathrm{~d} \phi \leqslant \\
& \leqslant \frac{2 m_{*} \cos ^{2}\left(m_{*}\right)-m_{*}}{\sin \left(m_{*}\right) \cos \left(m_{*}\right)} \int_{0}^{m_{*}} \frac{1}{\phi+m_{*}} \mathrm{~d} \phi \leqslant \ln 2<1,
\end{aligned}
$$

implying that the steady solution $\Phi_{0}$ is linearly stable.

For the case $S_{\mathrm{T}}<S_{\mathrm{B}}$ we again obtain $\lambda \leqslant \ln 2$, and hence, as to be expected, these steady states are also linearly stable.

\section{Saturation estimates}

\subsection{ESTIMATES THAT MAY INVOLVE TRANSIENT GROWTH}

Up to now we have estimates in terms of the matric flux potential perturbation $\varphi$. In this subsection we show that estimates for the saturation formulation can be obtained directly from the estimates derived in the previous section. 


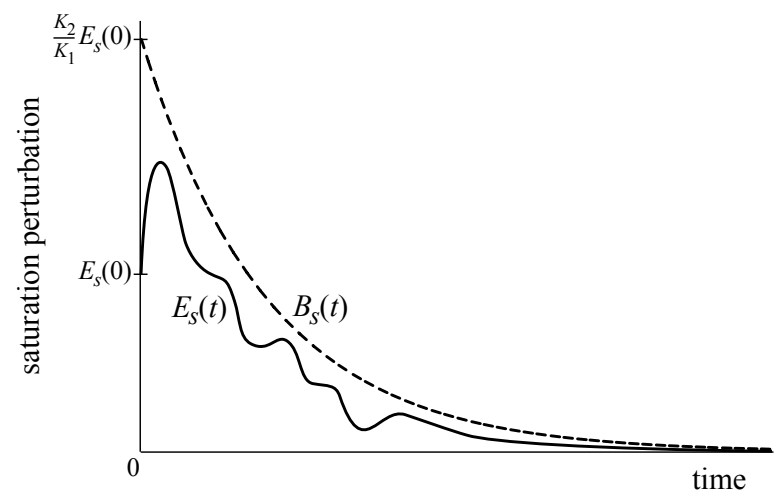

Figure 2. The norm gap may imply transient growth of $E_{S}(t)$, but $E_{S}(t)$ is nevertheless bounded by $B_{S}(t)$.

As for the matric flux potential $\Phi$, we write $S=S(x, y, z, t)$ in the form

$$
S=S_{0}(z)+s,
$$

where $s=s(x, y, z, t)$ vanishes at the top $(z=0)$ and bottom $(z=1)$ of the flow domain, and satisfies $\nabla s \cdot \mathbf{n}=0$ along $\partial \Omega_{\perp} \times(0,1)$, and where $S_{0}=f^{-1}\left(\Phi_{0}\right)$. The relation between the saturation perturbation $s$ and matric flux potential perturbation $\varphi$ follows from the expansion

$$
S=S_{0}(z)+s=S\left(\Phi_{0}+\varphi\right)=S\left(\Phi_{0}\right)+S^{\prime}\left(\Phi_{0}\right) \varphi+O\left(\varphi^{2}\right),
$$

which implies $s=S^{\prime}\left(\Phi_{0}\right) \varphi=\varphi / D\left(\Phi_{0}\right)$ in the linearized sense. Next we use (26) and (18) to obtain

$$
\begin{aligned}
\frac{1}{K_{2}} \int_{\Omega} s^{2}(t) & \leqslant \int_{\Omega} \frac{s^{2}(t)}{S^{\prime}\left(\Phi_{0}\right)} \leqslant \mathrm{e}^{-2(1-\lambda) t / K_{2}} \int_{\Omega} \frac{s^{2}(0)}{S^{\prime}\left(\Phi_{0}\right)} \leqslant \\
& \leqslant \frac{1}{K_{1}} \mathrm{e}^{-2(1-\lambda) t / K_{2}} \int_{\Omega} s^{2}(0),
\end{aligned}
$$

or

$$
E_{S}(t):=\int_{\Omega} s^{2}(t) \leqslant \frac{K_{2}}{K_{1}} \mathrm{e}^{-2(1-\lambda) t / K_{2}} \int_{\Omega} s^{2}(0)=: B_{s}(t) .
$$

Note that this estimate does not imply $\frac{\mathrm{d} E_{s}(t)}{\mathrm{d} t} \leqslant 0$. In fact, since $B_{s}(0)=\frac{K_{2}}{K_{1}} E_{S}(0)$ $\geqslant E_{s}(0)$, transient growth of $E_{s}(t)$ may occur as sketched in Figure 2. We discuss this in more detail in Section 7.

Remark 2. For Broadbridge-White soils we derive from $(\mathrm{I})_{4}$ that

$$
K_{1}=\frac{C-1}{C} \leqslant S^{\prime}\left(\Phi_{0}\right) \leqslant \frac{C}{C-1}=K_{2} .
$$


Using these estimates in (27) gives

$$
\int_{\Omega} s^{2}(t) \leqslant \frac{C^{2}}{(C-1)^{2}} \mathrm{e}^{-\frac{2(C-1)}{C}(1-\lambda) t} \int_{\Omega} s^{2}(0) .
$$

Note that for the limit $C \rightarrow \infty$ (Burgers soils), we obtain the sharp estimate

$$
\int_{\Omega} s^{2}(t) \leqslant \mathrm{e}^{-2(1-\lambda) t} \int_{\Omega} s^{2}(0) .
$$

This means that for Burgers soils $E_{s}(t)$ decreases monotonically in time and is bounded by an exponential decaying function.

For Gardner soils we obtain from (III) $)_{4}$ the estimates

$$
K_{1}=\frac{1}{2} \leqslant S^{\prime}\left(\Phi_{0}\right) \leqslant 1=K_{2} .
$$

Subtitution in (27) gives

$$
\int_{\Omega} s^{2}(t) \leqslant 2 \mathrm{e}^{-2(1-\lambda) t} \int_{\Omega} s^{2}(0) .
$$

Since $B_{s}(0)=2$, this is again not a sharp bound, and transient growth of $E_{s}(t)$ may occur.

\subsection{SHARP ESTIMATES}

In some cases a sharp estimate of the saturation perturbation can be obtained directly from (20). Substituting the relation $s=S^{\prime}\left(\Phi_{0}\right) \varphi$ gives the linearised perturbation equation

$$
\frac{\partial s}{\partial t}=\nabla \cdot\left(D\left(S_{0}\right) \nabla s\right)+\frac{\partial}{\partial z}\left\{D^{\prime}\left(S_{0}\right) \frac{\mathrm{d} S_{0}}{\mathrm{~d} z} s-R k^{\prime}\left(S_{0}\right) s\right\} .
$$

Note that this equation is more complex then Equation (20) for $\varphi$. This is the main reason why we choose the matric flux potential to study stability.

Multiplying (30) by $s$ and integrating over $\Omega$ gives

$$
\begin{aligned}
\frac{1}{2} \frac{\mathrm{d}}{\mathrm{d} t} \int_{\Omega} s^{2} & =-\int_{\Omega} D\left(S_{0}\right)|\nabla s|^{2}+\frac{1}{2} \int_{\Omega}\left(\frac{\mathrm{d}^{2}}{\mathrm{~d} z^{2}} D\left(S_{0}\right)-R \frac{\mathrm{d}}{\mathrm{d} z} k^{\prime}\left(S_{0}\right)\right) s^{2} \\
& =:-\int_{\Omega} D\left(S_{0}\right)|\nabla s|^{2}+I .
\end{aligned}
$$

The expression for $I$ can also be written as:

$$
I=\frac{1}{2} \int_{\Omega} \frac{\mathrm{d}}{\mathrm{d} S_{0}}\left(\frac{\mathrm{d} D\left(S_{0}\right)}{\mathrm{d} z}-R k^{\prime}\left(S_{0}\right)\right) \frac{\mathrm{d} S_{0}}{\mathrm{~d} z} s^{2} .
$$


Observe further that $\frac{\mathrm{d} D\left(S_{0}\right)}{\mathrm{d} z}=D^{\prime}\left(S_{0}\right) \frac{\mathrm{d} S_{0}}{\mathrm{~d} z}=\frac{D^{\prime}\left(S_{0}\right)}{D\left(S_{0}\right)} R\left(k\left(S_{0}\right)-F\right)$, where we have used (13). Hence

$$
\begin{aligned}
I=\frac{R}{2} \int_{\Omega}\left\{\frac{\mathrm{d}}{\mathrm{d} S_{0}}\left(\frac{D^{\prime}\left(S_{0}\right)}{D\left(S_{0}\right)}\left(k\left(S_{0}\right)-F\right)-k^{\prime}\left(S_{0}\right)\right)\right\} & \frac{\mathrm{d} S_{0}}{\mathrm{~d} z} s^{2}=: \\
= & : \frac{R}{2} \int_{\Omega} \gamma\left(S_{0}\right) \frac{\mathrm{d} S_{0}}{\mathrm{~d} z} s^{2} .
\end{aligned}
$$

Expanding $\gamma\left(S_{0}\right)$ in (32) and splitting the result into parts gives

$$
\gamma\left(S_{0}\right)=\gamma_{1}\left(S_{0}\right)+\gamma_{2}\left(S_{0}\right)
$$

with

$$
\begin{aligned}
& \gamma_{1}\left(S_{0}\right)=\frac{D^{\prime}\left(S_{0}\right)}{D\left(S_{0}\right)} k^{\prime}\left(S_{0}\right)-k^{\prime \prime}\left(S_{0}\right)=-D\left(S_{0}\right)\left(\frac{k^{\prime}\left(S_{0}\right)}{D\left(S_{0}\right)}\right)^{\prime}, \\
& \gamma_{2}\left(S_{0}\right)=\left(\frac{D^{\prime}\left(S_{0}\right)}{D\left(S_{0}\right)}\right)^{\prime}\left(k\left(S_{0}-F\right)\right) .
\end{aligned}
$$

For sharp estimates we want to have

$$
I \leqslant \mu \int_{\Omega}|\nabla s|^{2} \quad \text { with } \mu<\beta \quad \text { and } \beta:=\min _{S_{0}} D\left(S_{0}\right),
$$

since then, using the Poincaré inequality $-(\beta-\mu) \int_{\Omega}|\nabla s|^{2} \leqslant-(\beta-\mu) \int_{\Omega} s^{2}$ in (31), $\frac{\mathrm{d}}{\mathrm{d} t} \int_{\Omega} s^{2}<0$ and in particular

$$
\int_{\Omega} s^{2}(t) \leqslant \mathrm{e}^{-2(\beta-\mu) t} \int_{\Omega} s^{2}(0) \leqslant \int_{\Omega} s^{2}(0) .
$$

This would rule out transient growth as in Figure 2.

In Appendix B we show that for the Gardner class of soils $\mu \approx 0.9296$ and $\beta=1$. For the Broadbridge-White class of soils, however, we are not able to prove (34) for the range $1<C<2.5$. For the case $C \rightarrow \infty$, which corresponds to the Burgers class of soils, one can show, using techniques from Appendix $\mathrm{B}$, that $\mu \equiv 0$ and $\beta \equiv 1$.

For the Burgers class of soils we obtain even more than linear stability alone. For this particular case the nonlinear perturbation equation for $s$ is given by

$$
\frac{\partial s}{\partial t}=\Delta s-2 R \frac{\partial}{\partial z}\left(S_{0} s\right)-R \frac{\partial s^{2}}{\partial z} .
$$

Disregarding the quadratic term in (36) gives Equation (30). However, multiplying this quadratic term by $s$ and integration over $\Omega$ yields

$$
\int_{\Omega} \frac{\partial s^{2}}{\partial z} s=2 \int_{\Omega} s^{2} \frac{\partial s}{\partial z}=\frac{2}{3} \int_{\Omega} \frac{\partial s^{3}}{\partial z}=0
$$


implying that the steady solutions of the Burgers class of soils are nonlinearly (unconditionally) stable with respect to arbitrary finite-amplitude perturbations.

\section{Non-equilibrium Richards equation}

An extension of the Richards equation to take into account dynamic memory effects was suggested by Hassanizadeh and Gray $(1990 ; 1993)$. The key point in their model is the rejection of the equilibrium pressure-saturation relation. Instead they proposed:

$$
\left(\mathrm{RE}^{\prime}\right)\left\{\begin{array}{l}
\frac{\partial S}{\partial t}=\nabla \cdot\left(k(S) \nabla \widehat{\Psi}-R k(S) \mathbf{e}_{z}\right), \\
\tau \frac{\partial S}{\partial t}=\widehat{\Psi}-\Psi(S)
\end{array}\right.
$$

where $\widehat{\Psi}$ is the water pressure head and where $\tau$ is a dimensionless relaxation coefficient (taken as a positive constant). Combining the two equations in $\left(\mathrm{RE}^{\prime}\right)$ gives

$$
\frac{\partial S}{\partial t}=\nabla \cdot(D(S) \nabla S)+\tau \nabla \cdot\left(k(S) \nabla \frac{\partial S}{\partial t}\right)+R \frac{\partial}{\partial z} k(S)=0 .
$$

This equation will be referred to as the extended model.

Remark 3. The steady solutions of (37) do not depend on $\tau$. In fact, the steady equations are the same for both the standard Richards equation $(\mathrm{RE})_{2}$ and the extended model (37). So is the uniqueness result from Section 3.

In line with the general approach in Subsection 4.1, the normal procedure would be to rewrite (37) in terms of the matric flux potential $\Phi$ and consider the associated linearized perturbation equation. Rewriting (37) gives

$$
\frac{\partial S(\Phi)}{\partial t}=\Delta \Phi+\tau \nabla \cdot\left(\kappa(\Phi) \nabla \frac{\partial S(\Phi)}{\partial t}\right)+R \frac{\partial}{\partial z} \kappa(\Phi)=0 .
$$

Using the decomposition $\Phi=\Phi_{0}+\varphi$ in (38) and linearizing the resulting equation gives

$$
S^{\prime}\left(\Phi_{0}\right) \frac{\partial \varphi}{\partial t}=\Delta \varphi+\tau \nabla \cdot\left(\kappa\left(\Phi_{0}\right) \nabla\left\{S^{\prime}\left(\Phi_{0}\right) \frac{\partial \varphi}{\partial t}\right\}\right)+R \frac{\partial}{\partial z}\left(\kappa^{\prime}\left(\Phi_{0}\right) \varphi\right) .
$$

Following the variational approach, the $\tau$-term in (39) gives an expression of which the sign is not fixed. Therefore the matric flux potential $(\Phi)$ formulation is unsuitable for analyzing the stability of the $\left(\mathrm{RE}^{\prime}\right)$ steady states. 
To circumvent this problem, we consider the saturation perturbation equation related to $\left(\mathrm{RE}^{\prime}\right)$. In the linearized sense we find

$$
\frac{\partial s}{\partial t}=\nabla \cdot\left(D\left(S_{0}\right) \nabla s\right)+\tau \nabla \cdot\left(k\left(S_{0}\right) \nabla \frac{\partial s}{\partial t}\right)+\frac{\partial}{\partial z}\left\{D^{\prime}\left(S_{0}\right) \frac{\mathrm{d} S_{0}}{\mathrm{~d} z} s-R k^{\prime}\left(S_{0}\right) s\right\} .
$$

Multiplying (40) by $s$, integrating over $\Omega$, and collecting terms with a time derivative gives for any $\tau>0$

$$
\frac{1}{2} \frac{\mathrm{d}}{\mathrm{d} t}\left\{\int_{\Omega} s^{2}+\tau \int_{\Omega} k\left(S_{0}\right)|\nabla s|^{2}\right\}=-\int_{\Omega} D\left(S_{0}\right)|\nabla s|^{2}+I,
$$

where $I$ is given by (32). Now suppose that (34) holds. Then, with $\int_{\Omega} s^{2} \leqslant$ $\int_{\Omega}|\nabla s|^{2}$,

$$
\begin{aligned}
& \frac{1}{2} \frac{\mathrm{d}}{\mathrm{d} t}\left\{\int_{\Omega} s^{2}+\tau \int_{\Omega} k\left(S_{0}\right)|\nabla s|^{2}\right\} \leqslant-\int_{\Omega} D\left(S_{0}\right)|\nabla s|^{2}+\mu \int_{\Omega}|\nabla s|^{2} \leqslant \\
& \leqslant-(\beta-\mu) \int_{\Omega}|\nabla s|^{2} \leqslant-\frac{(\beta-\mu)}{2 \tau} \tau \int_{\Omega} k\left(S_{0}\right)|\nabla s|^{2}-\frac{(\beta-\mu)}{2} \int_{\Omega} s^{2} \leqslant \\
& \leqslant \mu^{*}\left\{\int_{\Omega} s^{2}+\tau \int_{\Omega} k\left(S_{0}\right)|\nabla s|^{2}\right\},
\end{aligned}
$$

where we have used that $k\left(S_{0}\right) \leqslant 1$ and where, since $\tau>0$,

$$
\mu^{*}=\max \left\{-\frac{\beta-\mu}{2 \tau},-\frac{\beta-\mu}{2}\right\}<0 .
$$

Integrating (41) gives

$$
\begin{aligned}
& \int_{\Omega} s^{2}(t) \leqslant \int_{\Omega} s^{2}(t)+\tau \int_{\Omega} k\left(S_{0}\right)|\nabla s(t)|^{2} \\
& \leqslant \mathrm{e}^{\mu^{*} t}\left\{\int_{\Omega} s^{2}(0)+\tau \int_{\Omega} k\left(S_{0}\right)|\nabla s(0)|^{2}\right\} .
\end{aligned}
$$

Since $\mu^{*}<0$, we obtain

$$
\int_{\Omega} s^{2}(t) \rightarrow 0 \quad \text { as } t \rightarrow \infty
$$

We conclude that once (34) is satisfied, linear stability of the steady solutions of both the standard and extended Richards equation is guaranteed. Estimates for $I$ were listed already in Section 5 following (34). 


\section{Different norms and transient growth}

In Sections 4 and 5 we considered linearized stability in terms of the (weighted) $L^{2}$-norms of the perturbations $\varphi$ of the matric flux potential and of the perturbations $s$ of the saturation. Independent of the chosen variable we obtained stability, but not necessarily time-monotonicity of the chosen norm. In this section we give an example of transient growth: initial growth of the norm of a perturbation which decays towards zero as $t \rightarrow \infty$, see sketch in Figure 2 . We consider Burgers soil for which the steady solution is unconditionally stable and for which the norms in terms of $\varphi$ and $s$ decay monotonically in time. We show below that in terms of the pressure head transient growth may occur.

For Burgers soils the relation between $S=\Phi$ and $\Psi$ is explicitly given by $\Psi(S)=1-S^{-1}$, or $S(\Psi)=\frac{1}{1-\Psi}$. We write $\Psi_{0}=\Psi\left(S_{0}\right)$ and redefine $k\left(\Psi_{0}\right):=$ $k\left(S\left(\Psi_{0}\right)\right), D\left(\Psi_{0}\right):=D\left(S\left(\Psi_{0}\right)\right)$ and $c\left(\Psi_{0}\right):=c\left(S\left(\Psi_{0}\right)\right)$. As in Section 4, we write $\Psi=\Psi_{0}(z)+\psi$, where $\psi=\psi(x, y, z, t)$ vanishes along the top and bottom of the flow domain. Linearizing $S(\Psi)$ gives the relation $s=S^{\prime}\left(\Psi_{0}\right) \psi=$ $\frac{1}{\left(1-\Psi_{0}\right)^{2}} \psi=S_{0}^{2} \psi$.

We consider the case $0<S_{\mathrm{B}}<S_{\mathrm{T}} \leqslant 1$. Using the relation between $s$ en $\psi$ in the saturation estimate (29) gives

$$
S_{\mathrm{B}}^{4} \int_{\Omega} \psi^{2}(t) \leqslant \int_{\Omega} s^{2}(t) \leqslant \mathrm{e}^{-2(1-\lambda) t} \int_{\Omega} s^{2}(0) \leqslant S_{\mathrm{T}}^{4} \mathrm{e}^{-2(1-\lambda) t} \int_{\Omega} \psi^{2}(0),
$$

or

$$
E_{\psi}(t):=\int_{\Omega} \psi^{2}(t) \leqslant\left(\frac{S_{\mathrm{T}}}{S_{\mathrm{B}}}\right)^{4} \mathrm{e}^{-2(1-\lambda) t} \int_{\Omega} \psi^{2}(0)=: B_{\psi}(t),
$$

with $\lambda \leqslant \ln 2$, see Section 4.2.1 and Remark 2. From (42) we observe that $B_{\psi}(0) \rightarrow E_{\psi}(0)$ as $S_{\mathrm{T}} / S_{\mathrm{B}} \rightarrow 1$ and therefore transient growth of $E_{\psi}(t)$ cannot occur for purely gravitational flows. However, for $S_{\mathrm{T}}>S_{\mathrm{B}}$ we can select a perturbation for which transient growth does occur.

To show this we consider the linearized perturbation equation for $\psi$. Using the relation between $s$ and $\psi$ in (30) and using the fact that $D\left(\Psi_{0}\right)=$ $k\left(\Psi_{0}\right) / c\left(\Psi_{0}\right)$ gives

$$
\begin{aligned}
\frac{\partial \psi}{\partial t}=\frac{k^{\prime}\left(\Psi_{0}\right)}{c\left(\Psi_{0}\right)} & {\left[2 \frac{\mathrm{d} \Psi_{0}}{\mathrm{~d} z}-R\right] \frac{\partial \psi}{\partial z}+\frac{k\left(\Psi_{0}\right)}{c\left(\Psi_{0}\right)} \Delta \psi-} \\
- & {\left[\frac{\left(k^{\prime}\left(\Psi_{0}\right)\right)^{2}}{k\left(\Psi_{0}\right) c\left(\Psi_{0}\right)}-\frac{k^{\prime \prime}\left(\Psi_{0}\right)}{c\left(\Psi_{0}\right)}\right]\left[\left(\frac{\mathrm{d} \Psi_{0}}{\mathrm{~d} z}\right)^{2}-R \frac{\mathrm{d} \Psi_{0}}{\mathrm{~d} z}\right] \psi }
\end{aligned}
$$

We restrict ourselves to $x, y$-periodic solutions $\psi=\psi(z, t) \mathrm{e}^{i \mathbf{k} \cdot \mathbf{x}}$, where $\mathbf{k}=$ $\left(k_{x}, k_{y}\right)^{T}$ denotes the wave vector and $\mathbf{x}=(x, y)^{T}$. Using $k(\Psi)$ and $c(\Psi)$ in 

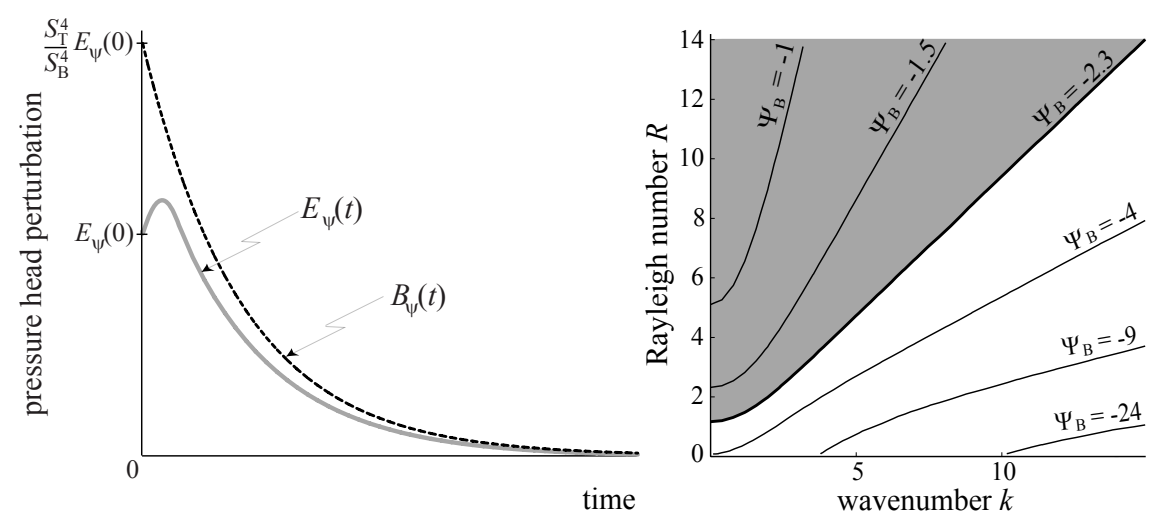

Figure 3. Left: a typical picture of the norm $E_{\psi}(t)$. Note that $E_{\psi}^{\prime}(0)=\sigma_{\max }>0$. Right: neutral curves (i.e. $\sigma \equiv 0$ ) corresponding to eigenvalue problem (47). The shaded region corresponds to the case $\Psi_{\mathrm{T}}=0, \Psi_{\mathrm{B}}=-2.3\left(S_{\mathrm{T}}=1, S_{\mathrm{B}}=0.3\right)$ and depicts the region where $\sigma_{\max }>0$. The white region corresponds to $\sigma_{\max }<0$.

(43) gives for the amplitude $\psi=\psi(z, t)$

$$
\frac{\partial \psi}{\partial t}=\frac{\partial^{2} \psi}{\partial z^{2}}-k^{2} \psi+\mathcal{A}_{1}\left(\Psi_{0}\right) \frac{\partial \psi}{\partial z}+\mathcal{A}_{2}\left(\Psi_{0}\right) \psi=: \mathcal{A}\left(\Psi_{0}\right) \psi
$$

where $k=|\mathbf{k}|$ and

$$
\mathcal{A}_{1}\left(\Psi_{0}\right)=\frac{4 \Psi_{0}^{\prime}(z)-2 R}{1-\Psi_{0}} \quad \text { and } \quad \mathcal{A}_{2}\left(\Psi_{0}\right)=\frac{2\left(\Psi_{0}^{\prime}(z)\right)^{2}-2 R \Psi_{0}^{\prime}(z)}{\left(1-\Psi_{0}\right)^{2}} .
$$

Multiplying Equation (44) by $\psi$ and integrating the result gives

$$
\frac{1}{2} \frac{\mathrm{d} E_{\psi}(t)}{\mathrm{d} t}:=\frac{1}{2} \frac{\mathrm{d}}{\mathrm{d} t} \int_{0}^{1} \psi^{2}(t) \mathrm{d} z=\int_{0}^{1}\left(\mathcal{A}\left(\Psi_{0}\right) \psi(t)\right) \psi(t) \mathrm{d} z
$$

Transient growth is said to occur if $E_{\psi}(t)>E_{\psi}(0)$ for some $t>0$. This happens, for instance, if $\left.\frac{1}{E_{\psi}(0)} \frac{\mathrm{d} E_{\psi}(t)}{\mathrm{d} t}\right|_{t=0}>0$, see Figure 3 (left). To find the maximal initial growth we use (45) and consider

$$
\left.\frac{1}{2} \frac{1}{E_{\psi}(0)} \frac{\mathrm{d} E_{\psi}(t)}{\mathrm{d} t}\right|_{t=0}=\int_{0}^{1}\left(\mathcal{A}\left(\Psi_{0}\right) \psi(0)\right) \psi(0) \mathrm{d} z / \int_{0}^{1} \psi^{2}(0) \mathrm{d} z
$$

This leads to the maximum problem

$$
\sigma_{\max }=\sup _{\psi(0) \neq 0}\left\{\int_{0}^{1}\left(\mathcal{A}\left(\Psi_{0}\right) \psi(0)\right) \psi(0) \mathrm{d} z / \int_{0}^{1} \psi^{2}(0) \mathrm{d} z\right\} .
$$

Farrell and Ioannou (1996), among others, showed that the normalized maximal initial slope $\sigma_{\max }$ and the initial perturbation $\psi(0)$ producing this initial 
growth are found from the eigenanalysis of the symmetric part of operator $\mathcal{A}\left(\Psi_{0}\right)$, i.e.

$$
\frac{1}{2}\left(\mathcal{A}\left(\Psi_{0}\right)+\mathcal{A}^{*}\left(\Psi_{0}\right)\right) \psi(0)=\sigma \psi(0),
$$

where * denotes the adjoint operator with respect to the $L^{2}$-norm. In particular, the maximal positive eigenvalue and its associated eigenfunction provide the initial slope of $E_{\psi}(t)$ and the initial perturbation.

Remark 4. The estimates obtained in Sections 4 and 5 imply that the eigenvalues of $\mathcal{A}\left(\Psi_{0}\right)$ all have negative real parts. Hence if $\mathcal{A}\left(\Psi_{0}\right)$ were a selfadjoint operator, then all (real) eigenvalues of (47) would have been negative as well and no transient growth would occur, see also Reddy and Henningson (1993).

We solved (47) numerically for fixed $\Psi_{\mathrm{T}} \equiv 0$ and various $-\infty<\Psi_{\mathrm{B}}<0$, and for Rayleigh numbers $R$ and wavenumbers $k$ in relevant ranges. The result is shown in Figure 3 (right). Given the boundary conditions $\Psi_{\mathrm{T}}, \Psi_{\mathrm{B}}$, and the Rayleigh number $R$, we can distinguish grey regions where $\sigma_{\max }>0$, indicating initial transient growth, and white regions where $\sigma_{\max }<0$. We do note here that since $E_{\psi}(t)$ is bounded by $B_{\psi}(t)$, the growth of the norm is only a transient phenomenon because $B_{\psi}(t) \rightarrow 0$ as $t \rightarrow \infty$.

\section{Discussion}

Kapoor (1996) derived stability criteria for the various types of steady vertical upward and downward flows in homogeneous, unsaturated soils. These criteria are summarized in Figure 4. Based on experimental evidence that observed fingers often are long and narrow, he assumed that the vertical length scale of the perturbations is large compared to the horizontal length scale and on that basis he simplified the linearized equation for the perturbation of the suction head. Allowing for the sign changes in going from the suction head to the pressure head, Kapoor (1996) in effect ignored in the right hand side of equation (43) the entire first term and $z$-dependent part of the second term. The results shown in Figure 4 then follow from considering the sign of the coefficient of $\psi$ in the last term.

Linear stability analysis concerns the process of initiation of the fingers and in that stage the vertical length scale of the perturbations is still small. Therefore we reconsidered the stability of steady vertical flows, without ignoring the vertical gradients. First, we proved the uniqueness of the solutions for steady vertical flows. Subsequently we used the so-called energy method to prove the stability of the steady flows for various classes of soils and derived estimates of the rate of decay of perturbations. The decay of the 


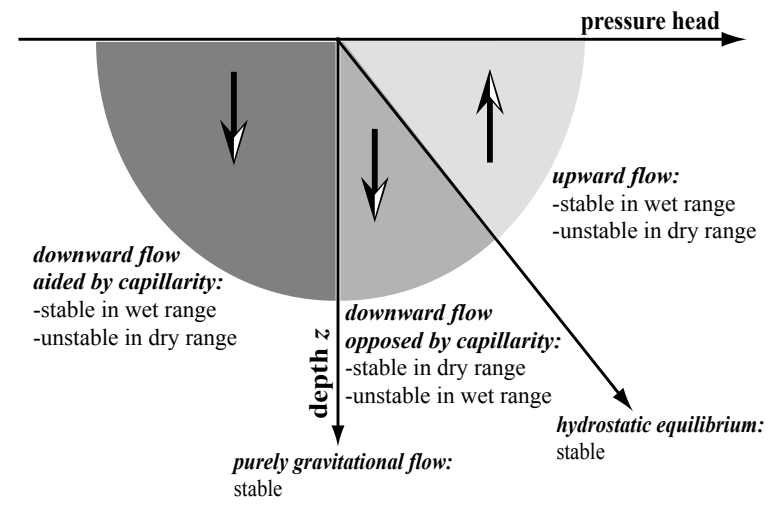

Figure 4. Overview of Kapoor's stability results.

perturbation is proportional to $\exp \left(-2(1-\lambda) D_{\min } t\right)$, where $D_{\min }$ is an appropriate minimum value of the diffusivity. As is to be expected, an increase of $D_{\min }$ or a decrease of $H$ speeds up the decay. The analysis is complicated by the fact that, depending on the norm used in the analysis, the ultimate decay may be preceded by transient growth. Contrary to Kapoor's hypothesis, our analysis shows that the vertical gradients play an essential stabilizing role. In the analysis we considered several classes of soils, spanning a wide range of soil properties. For the Burgers class of soils, we were able to show that the steady solutions are nonlinearly stable with respect to arbitrary finiteamplitude perturbations. Finally, we showed that for the Richards equation extended with a term accounting for dynamic memory effects steady flows also remain stable.

\section{Appendix A. Proof of Theorem 1}

Since $(\mathrm{P} 1)_{1}$ is in divergence form, we follow Gilbarg and Trudinger (1977) to prove a comparison result and uniqueness. For technical reasons we extend the domain of definition of $\kappa$. Let

$$
\widehat{\kappa}(\Phi)= \begin{cases}0 & \text { for } \quad \Phi<0, \\ \kappa(\Phi) & \text { for } \quad 0 \leqslant \Phi \leqslant M, \\ \kappa(M)=1 & \text { for } \Phi \geqslant M .\end{cases}
$$

The function $\widehat{\kappa}$ is clearly uniformly Lipschitz continuous on $\mathbb{R}$. Now suppose $\Phi_{1}$ and $\Phi_{2}$ are two solutions of Problem (P1), with ordered boundary data: i.e.

$$
\Phi_{1} \geqslant \Phi_{2} \quad \text { at } \Omega_{\perp} \times(\{0\} \cup\{1\}) .
$$


We show below that

$$
\Phi_{1} \geqslant \Phi_{2} \quad \text { in } \bar{\Omega} .
$$

Let $\delta>0$ (fixed) and set

$$
\varphi=\frac{w}{\delta+w}, \quad w=\left(\Phi_{1}-\Phi_{2}-\delta\right)_{+} \geqslant 0,
$$

where $(u)_{+}=\max \{u, 0\}$. We test $(\mathrm{P} 1)_{1}$ for the difference $\Phi_{1}-\Phi_{2}$ with $\varphi$ :

$$
\int_{\Omega}\left\{\nabla\left(\Phi_{1}-\Phi_{2}\right)-R\left(\widehat{\kappa}\left(\Phi_{1}\right)-\widehat{\kappa}\left(\Phi_{2}\right)\right) \mathbf{e}_{z}\right\} \cdot \nabla \varphi=0
$$

This gives

$$
\delta \int_{\left\{\Phi_{1}-\Phi_{2}>\delta\right\}} \frac{|\nabla w|^{2}}{(\delta+w)^{2}}-\delta R \int_{\left\{\Phi_{1}-\Phi_{2}>\delta\right\}} \frac{\partial_{z} w}{(\delta+w)^{2}}\left(\widehat{\kappa}\left(\Phi_{1}\right)-\widehat{\kappa}\left(\Phi_{2}\right)\right)=0 .
$$

Since

$$
\begin{aligned}
& R \int_{\left\{\Phi_{1}-\Phi_{2}>\delta\right\}} \frac{\partial_{z} w}{(\delta+w)^{2}}\left(\widehat{\kappa}\left(\Phi_{1}\right)-\widehat{\kappa}\left(\Phi_{2}\right)\right) \leqslant \\
& \quad \leqslant \frac{1}{2} \int_{\left\{\Phi_{1}-\Phi_{2}>\delta\right\}} \frac{|\nabla w|^{2}}{(\delta+w)^{2}}+\frac{R^{2}}{2} \int_{\left\{\Phi_{1}-\Phi_{2}>\delta\right\}} \frac{\left(\widehat{\kappa}\left(\Phi_{1}\right)-\widehat{\kappa}\left(\Phi_{2}\right)\right)^{2}}{(\delta+w)^{2}},
\end{aligned}
$$

and $\delta+w=\Phi_{1}-\Phi_{2}$ on $\left\{\Phi_{1}-\Phi_{2}-\delta>0\right\}$, we find

$$
\int_{\left\{\Phi_{1}-\Phi_{2}>\delta\right\}} \frac{|\nabla w|^{2}}{(\delta+w)^{2}} \leqslant R^{2} \int_{\left\{\Phi_{1}-\Phi_{2}>\delta\right\}} \frac{\left(\widehat{\kappa}\left(\Phi_{1}\right)-\widehat{\kappa}\left(\Phi_{2}\right)\right)^{2}}{\left(\Phi_{1}-\Phi_{2}\right)^{2}} .
$$

Since $\widehat{\kappa}$ is Lipschitz-continuous with constant $L$, we have

$$
\int_{\left\{\Phi_{1}-\Phi_{2}>\delta\right\}} \frac{|\nabla w|^{2}}{(\delta+w)^{2}} \leqslant R^{2} L^{2} \operatorname{meas}(\Omega) .
$$

Next we apply the Poincaré inequality to

$$
\left|\ln \left(1+\frac{w}{\delta}\right)\right|=|\ln (\delta+w)-\ln (\delta)|
$$

and obtain that there exists $K>0$ such that

$$
\int_{\Omega}\left|\ln \left(1+\frac{w}{\delta}\right)\right|^{2} \leqslant K \quad(\text { for all } \delta>0) .
$$

Letting $\delta \rightarrow 0$, we see that $w$ must vanish in $\Omega$, that is $\Phi_{1} \leqslant \Phi_{2}$. Since

$$
0=f(0) \leqslant \Phi \leqslant f(1)=M,
$$


and since constants satisfy the equation, we immediately deduce for any solution of $(\mathrm{P} 1)$

$$
0 \leqslant \Phi \leqslant M \quad \text { in } \bar{\Omega} .
$$

As a consequence we can replace $\widehat{\kappa}$ by $\kappa$. Now suppose $\Phi_{1}$ and $\Phi_{2}$ are two solutions of (P1) for the same boundary data. The above comparison argument then implies both $\Phi_{1} \leqslant \Phi_{2}$ and $\Phi_{1} \geqslant \Phi_{2}$ in $\bar{\Omega}$. Hence $\Phi_{1}=\Phi_{2}$ in $\bar{\Omega}$.

\section{Appendix B. Saturation estimates for Gardner class soils}

First we evaluate the components $\gamma_{1}\left(S_{0}\right)$ and $\gamma_{2}\left(S_{0}\right)$ of $\gamma\left(S_{0}\right)$. This can be done by direct lengthy computation, using the expressions (II) ${ }_{1}$ for $k\left(S_{0}\right)$ and (II) $)_{2}$ for $D\left(S_{0}\right)$ in (33a) and (33b). A simpler procedure is to observe that (II) $)_{1,2}$ imply $k^{\prime}\left(S_{0}\right)=\left(D\left(S_{0}\right)-1\right) D\left(S_{0}\right)$ and $D^{\prime}\left(S_{0}\right)=\left(2-4 k\left(S_{0}\right)\right) D\left(S_{0}\right)$ so that the expressions for $\gamma_{1}\left(S_{0}\right)$ and $\gamma_{2}\left(S_{0}\right)$ reduce to

$$
\begin{aligned}
& \gamma_{1}\left(S_{0}\right)=-D\left(S_{0}\right) D^{\prime}\left(S_{0}\right)=-\frac{1}{2}\left(D^{2}\left(S_{0}\right)\right)^{\prime}, \\
& \gamma_{2}\left(S_{0}\right)=4 k^{\prime}\left(S_{0}\right)\left(F-k\left(S_{0}\right)\right) .
\end{aligned}
$$

We need to estimate

$$
I=\frac{R}{2} \int_{\Omega} \gamma\left(S_{0}\right) \frac{\mathrm{d} S_{0}}{\mathrm{~d} z} s^{2}=\frac{R}{2} \int_{\Omega}\left(\gamma_{1}\left(S_{0}\right)+\gamma_{2}\left(S_{0}\right)\right) \frac{\mathrm{d} S_{0}}{\mathrm{~d} z} s^{2} .
$$

We first consider the downward flow case, i.e. $F>k\left(S_{\mathrm{T}}\right) \geqslant k\left(S_{0}\right)$ for every $0 \leqslant S_{\mathrm{B}} \leqslant S_{0}<S_{\mathrm{T}} \leqslant 1$. Since $\frac{\mathrm{d} S_{0}}{\mathrm{~d} z}$ is negative, we want to show that $\gamma\left(S_{0}\right)$ is positive for all $R>0$. From (48a) and (48b) it follows that $\gamma_{2}\left(S_{0}\right)>0$ and

$$
\gamma_{1}\left(S_{0}\right)= \begin{cases}<0 & \text { for } 0 \leqslant S_{\mathrm{B}}<S_{0}<\frac{1}{2}, \\ >0 & \text { for } \frac{1}{2}<S_{0}<S_{\mathrm{T}} \leqslant 1\end{cases}
$$

We distinguish the following cases:

(1) $0 \leqslant S_{\mathrm{B}}<\frac{1}{2} \leqslant S_{\mathrm{T}} \leqslant 1$. Let $z^{*}$ be such that $S_{0}\left(z^{*}\right)=\frac{1}{2}$. Then $S_{\mathrm{B}} \leqslant S_{0}<\frac{1}{2}$ for $z \in\left(z^{*}, 1\right]$, see also the construction in Figure 5(a). Hence, with (50) and inequality (24), we obtain

$$
\begin{aligned}
I & \leqslant \frac{R}{2} \int_{\Omega^{*}} \gamma_{1}\left(S_{0}\right) \frac{\mathrm{d} S_{0}}{\mathrm{~d} z} s^{2}=\frac{R}{4} \int_{\Omega^{*}}\left(D^{2}\left(S_{0}\right)\right)^{\prime}\left(-\frac{\mathrm{d} S_{0}}{\mathrm{~d} z}\right) s^{2}= \\
& =-\frac{R}{4} \int_{\Omega^{*}} \frac{\mathrm{d}}{\mathrm{d} z}\left(D^{2}\left(S_{0}\right)\right) s^{2} \leqslant \\
& \leqslant\left(-\frac{R}{4} \int_{z^{*}}^{1} \frac{\mathrm{d}}{\mathrm{d} z}\left(D^{2}\left(S_{0}\right)\right)(1-z) \mathrm{d} z\right) \int_{\Omega}|\nabla s|^{2}=: \mu \int_{\Omega}|\nabla s|^{2} .
\end{aligned}
$$



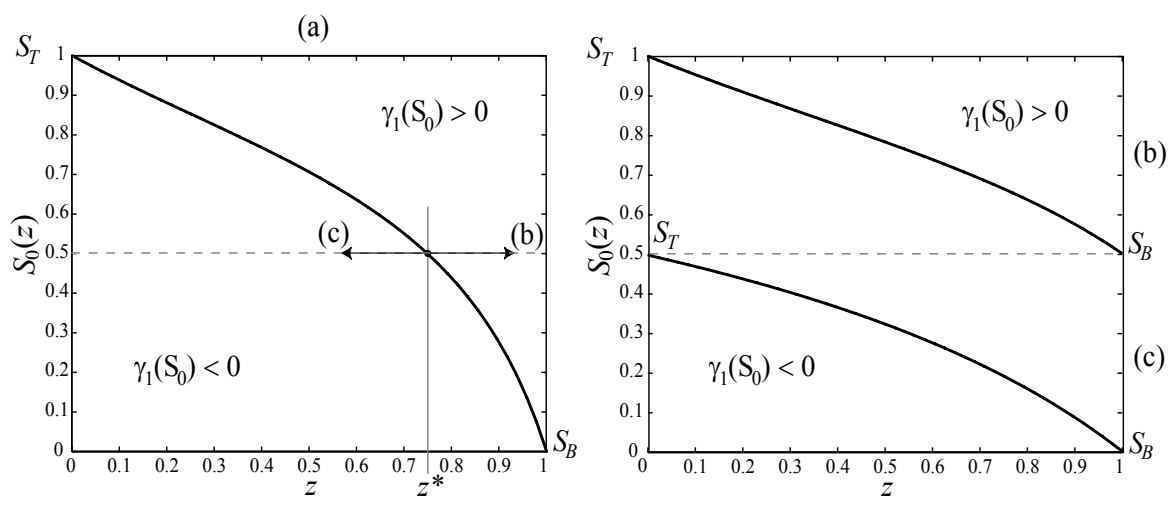

Figure 5. Definition of the point $z^{*}$ for (a): $S_{\mathrm{T}}=1$ and $S_{\mathrm{B}}=0$, (b): $S_{\mathrm{T}}=1, S_{\mathrm{B}}=\frac{1}{2}, z^{*}=1$, and (c): $S_{\mathrm{T}}=\frac{1}{2}, S_{\mathrm{B}}=0, z^{*}=0$.

Integration by parts gives for $\mu$

$$
\begin{aligned}
\mu & =\left[-\frac{R}{4} D^{2}\left(S_{0}(z)\right)(1-z)\right]_{z^{*}}^{1}-\frac{R}{4} \int_{z^{*}}^{1} D^{2}\left(S_{0}(z)\right) \mathrm{d} z= \\
& =\frac{R}{4} \int_{z^{*}}^{1}\left[D^{2}\left(\frac{1}{2}\right)-D^{2}\left(S_{0}(z)\right)\right] \mathrm{d} z= \\
& =\frac{1}{4} \int_{S_{\mathrm{B}}}^{\frac{1}{2}} \frac{D^{2}\left(\frac{1}{2}\right)-D^{2}\left(S_{0}\right)}{F-k\left(S_{0}\right)} D\left(S_{0}\right) \mathrm{d} S_{0} .
\end{aligned}
$$

The factor $\mu$ in (51) can be estimated by using $F>k\left(S_{\mathrm{T}}\right) \geqslant k\left(\frac{1}{2}\right)=\frac{1}{2}$. This gives, using $D^{\prime}(S)=2(1-2 S) D^{2}(S)$ and $\left(D\left(\frac{1}{2}\right)-D(S)\right) /\left(k\left(\frac{1}{2}\right)-\right.$ $k(S))=2(1-2 S)$,

$$
\begin{aligned}
\mu & <\frac{1}{4} \int_{S_{\mathrm{B}}}^{\frac{1}{2}} \frac{D^{2}\left(\frac{1}{2}\right)-D^{2}\left(S_{0}\right)}{k\left(\frac{1}{2}\right)-k\left(S_{0}\right)} D\left(S_{0}\right) \mathrm{d} S_{0} \leqslant \\
& \leqslant \frac{1}{4} \int_{0}^{\frac{1}{2}} 2\left(1-2 S_{0}\right)\left(2+D\left(S_{0}\right)\right) D\left(S_{0}\right) \mathrm{d} S_{0}= \\
& \leqslant \frac{1}{2} \int_{0}^{\frac{1}{2}} \frac{D^{\prime}\left(S_{0}\right)}{D\left(S_{0}\right)} \mathrm{d} S_{0}+\frac{1}{4} \int_{0}^{\frac{1}{2}} D^{\prime}\left(S_{0}\right) \mathrm{d} S_{0}=\frac{1}{2}\left(\ln 2+\frac{1}{2}\right)=: \mu_{1},
\end{aligned}
$$

with $\mu_{1} \approx 0.5966$.

(2) $S_{\mathrm{B}}<S_{\mathrm{T}}<\frac{1}{2}$. For this case we have $z^{*}=0$, see Figure 5(c). Again we obtain for $\mu$ an expression similar to (51):

$$
\mu=\frac{1}{4} \int_{S_{\mathrm{B}}}^{S_{\mathrm{T}}} \frac{D^{2}\left(S_{\mathrm{T}}\right)-D^{2}\left(S_{0}\right)}{F-k\left(S_{0}\right)} D\left(S_{0}\right) \mathrm{d} S_{0} .
$$




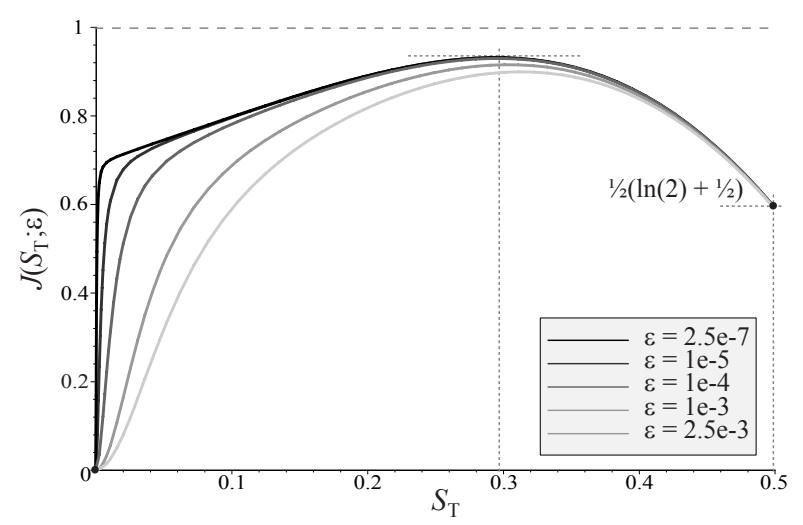

Figure 6. Plot of $J\left(S_{\mathrm{T}} ; \varepsilon\right)$ for $0 \leqslant S_{\mathrm{T}} \leqslant \frac{1}{2}$ and several values of $\varepsilon$. The maximum is located at $S_{\mathrm{T}} \approx 0.2952$, and its value is given by 0.9296 .

For a given $S_{\mathrm{T}}, S_{\mathrm{B}}$ and $R>0$, there exists a $\varepsilon>0$ such that $F=k\left(S_{\mathrm{T}}\right)+\varepsilon$. Hence

$$
\mu=\frac{1}{4} \int_{0}^{S_{\mathrm{T}}} \frac{D^{2}\left(S_{\mathrm{T}}\right)-D^{2}\left(S_{0}\right)}{k\left(S_{\mathrm{T}}\right)+\varepsilon-k\left(S_{0}\right)} D\left(S_{0}\right) \mathrm{d} S_{0}=: J\left(S_{\mathrm{T}} ; \varepsilon\right) .
$$

Since we could not find a closed form expression for $J\left(S_{\mathrm{T}} ; \varepsilon\right)$, we evaluate it numerically. This is rather straight forward since the presence of $\varepsilon>0$ makes the integral nonsingular. Note that $J\left(S_{\mathrm{T}} ; \varepsilon_{1}\right)<J\left(S_{\mathrm{T}} ; \varepsilon_{2}\right)$ when $\varepsilon_{1}>\varepsilon_{2}$ for every $0<S_{\mathrm{T}}<\frac{1}{2}$. Maximizing $J\left(S_{\mathrm{T}} ; \varepsilon\right)$ for $0<S_{\mathrm{T}}<\frac{1}{2}$ and $\varepsilon \downarrow 0$ gives $\mu<0.9296=: \mu_{2}$ (see Figure 6). For the derivation of this upperbound for $\mu$, we have only used the soil properties $D\left(S_{0}\right)$ and $k\left(S_{0}\right)$. As a consequence, this upperbound is rather crude.

(3) $\frac{1}{2} \leqslant S_{\mathrm{B}}<S_{\mathrm{T}} \leqslant 1$. Now we have $z^{*}=1$, see Figure 5 (b). This case is trivial since $\gamma_{1}\left(S_{0}\right)>0$ for every $\frac{1}{2} \leqslant S_{\mathrm{B}}<S_{0} \leqslant S_{\mathrm{T}} \leqslant 1$.

Remark 5. The case $S_{\mathrm{T}} \leqslant S_{\mathrm{B}}$ is treated in a similar way. Now $\frac{\mathrm{d} S_{0}}{\mathrm{~d} z} \geqslant 0$ and $F<k\left(S_{\mathrm{T}}\right)$. Hence $\gamma_{2}\left(S_{0}\right) \frac{\mathrm{d} S_{0}}{\mathrm{~d} z}<0$. Further,

$$
\gamma_{1}\left(S_{0}\right)= \begin{cases}<0 & \text { for } 0 \leqslant S_{\mathrm{T}}<S_{0}<\frac{1}{2} \\ >0 & \text { for } \frac{1}{2}<S_{0}<S_{\mathrm{B}} \leqslant 1\end{cases}
$$

This implies a repetition of the derivation where now $S_{\mathrm{T}}$ is replaced by $S_{\mathrm{B}}$ and vice versa. It gives the same estimates for $\mu$. 
Finally, let $\bar{\mu}=\max \left\{\mu_{1}, \mu_{2}\right\}=0.9296$. Using $\bar{\mu}$ and the fact that $\beta=1$ in (35), we obtain

$$
\int_{\Omega} s^{2} \leqslant \mathrm{e}^{-2(1-\bar{\mu}) t} \int_{\Omega} s^{2}(0) \leqslant \int_{\Omega} s^{2}(0),
$$

with $-2(1-\bar{\mu}) \approx-0.1408$.

\section{References}

Broadbridge, P. and I. White: 1988, 'Constant Rate Rainfall Infiltration: A Versatile Nonlinear Model I. Analytic Solution’. Water Resour. Res. 24, 145-154.

Chuoke, R. L., P. van Meurs, and C. van der Poel: 1959, 'The Instability of Slow, Immiscible, Viscous Liquid-Liquid Displacements in Porous Media'. Petroleum Transactions of the American Institute of Mining Engineering 216, 188-194.

Clothier, B. E., J. H. Knight, and I. White: 1981, 'Burgers' Equation: Application to Field Constant-Flux Infiltration'. Soil Sci. 132, 252-261.

de Rooij, G. H.: 2000, 'Modeling Fingered Flow of Water in Soils Owing to Wetting Front Instability: A Review'. Journal of Hydrology 231-232, 277-294.

Diment, G. A. and K. K. Watson: 1982, 'Stability Analysis of Water Movement in Unsaturated Porous Materials: 1. Theoretical Considerations'. Water Resources Res. 18(2), 1248-1254.

Diment, G. E. and K. K. Watson: 1983, 'Stability Analysis of Water Movement in Unsaturated Porous Materials: 2. Numerical Studies'. Water Resour. Res. 19, 1002-1010.

Diment, G. E. and K. K. Watson: 1985, 'Stability Analysis of Water Movement in Unsaturated Porous Materials: 3. Experimental Studies'. Water Resour. Res. 21(7), 979-984.

Egorov, A. G., R. Z. Dautov, J. L. Nieber, and A. Y. Sheshukov: 2002, 'Stability Analysis of Traveling Wave Solution for Gravity-Driven Flow'. In: S. M. Hassanizadeh, R. J. Schotting, W. G. Gray, and G. F. Pinder (eds.): Computational Methods in Water Resources, Vol. 1. Amsterdam, pp. 121-128, Elsevier.

Egorov, A. G., R. Z. Dautov, J. L. Nieber, and A. Y. Sheshukov: 2003, 'Stability Analysis of Gravity-Driven Infiltrating Flow'. Accepted for publication in Water Resour. Res.

Engelberts, W. F. and L. J. Klinkenberg: 1951, 'Laboratory Experiments on the Displacement of Oil by Water from Packs of Granular Material'. In: Proceedings 3rd World Petroleum Congress, The Hague, Vol. 2. pp. 544-554.

Farrell, B. F. and P. J. Ioannou: 1996, 'Generalized Stability Theory. Part I: Autonomous Operators'. J. Atmos. Sci. 53, 2025-2040.

Gardner, W. R.: 1958, 'Some Steady-State Solutions of the Unsaturated Moisture Flow Equation with Application to Evaporation from a Water Table'. Soil Sci. 85, 228-232.

Gilbarg, D. and N. S. Trudinger: 1977, Elliptic Partial Differential Equations of Second Order. Berlin: Springer-Verlag.

Hassanizadeh, S. M. and W. G. Gray: 1990, 'Mechanics and Thermodynamics of Multiphase Flow in Porous Media Including Interphase Boundaries'. Adv. Water Resour. 13, 169-186.

Hassanizadeh, S. M. and W. G. Gray: 1993, 'Thermodynamic Basis of Capillary Pressure in Porous Media'. Water Resources Res. 29, 3389-3405.

Hendrickx, J. M. H. and M. Flury: 2001, 'Uniform and Preferential Flow Mechanisms in the Vadose Zone'. In: E. Commission on Geosciences and R. (CGER) (eds.): Conceptual Models of Flow and Transport in the Fractured Vadose Zone. Washington, DC, pp. 149188, National Academy Press.

Hill, D. E. and J.-Y. Parlange: 1972, 'Wetting Front Instability in Homogeneous Soils'. Soil Sci. Soc. Am. Proc. 36, 697-702. 
Hill, S.: 1952, 'Channelling in Packed Columns'. Chemical Engineering Science 1, 247-253. Kapoor, V.: 1996, 'Criterion for Instability of Steady-State Unsaturated Flows'. Transport in Porous Media 25, 335-350.

Kirchhoff, G.: 1894, Vorlesungen über Die Theorie der Wärme, Herausgegeben Von M. Planck. Leipzig: Teubner.

Miller, E. E. and R. D. Miller: 1956, 'Physical Theory of Capillary Flow Phenomena'. J. Appl. Phys. 27, 324-332.

Parlange, J.-Y. and D. E. Hill: 1976, 'Theoretical Analysis of Wetting Front Instability in Soils'. Soil Sci. 122, 236-239.

Parlange, J.-Y., T. S. Steenhuis, L. Li, D. A. Barry, and F. Stagnitti: 2002, 'Column Flow in Stratified Soils and Fingers in Hele-Shaw Cells: A Review'. In: P. A. C. Raats, D. Smiles, and A. W. Warrick (eds.): Environmental Mechanics. Water, Mass and Energy Transfer in the Biosphere. Washington, DC and CSIRO, Australia: American Geophysical Union, pp. $79-85$.

Philip, J. R.: 1975a, 'The Growth of Disturbances in Unstable Infiltration Flows'. Soil Sci. Soc. Am. Proc. 39, 1049-1053.

Philip, J. R.: 1975b, 'Stability Analysis of Infiltration'. Soil Sci. Soc. Am. Proc. 39, 1042-1049.

Raats, P. A. C.: 1973, 'Steady Upward and Downward Flows in a Class of Unsaturated Soils'. Soil Sci. 115(6), 409-413.

Raats, P. A. C.: 1984, 'Applications of the Theory of Mixtures in Soil Science'. In: C. Truesdell (ed.): Rational Thermodynamics, with an Appendix by C. - C. Wang. New York: SpringerVerlag, Chapt. Appendix 5D, pp. 326-343.

Reddy, S. C. and D. S. Henningson: 1993, 'Energy Growth in Viscous Channel Flows'. J. Fluid Mech. 252, 209-238.

Richards, L. A.: 1931, 'Capillary Conduction of Liquids Through Porous Mediums'. Physics 1, 318-333.

Saffman, P. G. and S. G. Taylor: 1958, 'The Penetration of a Fluid Into a Porous Medium or Hele-Shaw Cell Containing a More Viscous Liquid'. Royal Society of London Proceedings A245, 312-329.

Straughan, B.: 1992, The Energy Method, Stability, and Nonlinear Convection. New York: Springer-Verlag.

Tabuchi, T.: 1961, 'Infiltration and Ensuing Percolation in Columns of Layered Glass Particles Packed in Laboratory'. Nogyo Dobuku Kenkyu, Bessatu (Transactions Agricultural Engineering Society, Japan) 2, 27-36.

Whitaker, S.: 1986, 'Flow in Porous Media II: The Governing Equations for Immiscible, TwoPhase Flow'. Transport in Porous Media 1, 105-125.

Zeidler, E.: 1995, Applied Functional Analysis, Vol. 108, 109 of Applied Mathematical Sciences. New York: Springer-Verlag. 